\title{
RUNGE-KUTTA METHODS APPLIED TO FULLY IMPLICIT DIFFERENTIAL-ALGEBRAIC EQUATIONS OF INDEX 1
}

\author{
ANNE KVAERNØ
}

\begin{abstract}
In this paper we study the order of Runge-Kutta methods applied to differential-algebraic equations of index one. We derive general order conditions for the local order $k_{L}$, and give a convergence result, which shows that the order $k_{G}$ of the global error satisfies $k_{G} \geq k_{L}-1$. We also describe some numerical experiments, which are in agreement with our results.
\end{abstract}

\section{INTRODUCTION}

A general differential-algebraic equation (DAE) has the form

$$
F\left(v, v^{\prime}, x\right)=0
$$

with initial values

$$
v\left(x_{0}\right)=v_{0}, \quad v^{\prime}\left(x_{0}\right)=v_{0}^{\prime},
$$

where $v: \mathbf{R} \rightarrow \mathbf{R}^{m-1}$ and $F: \mathbf{R}^{m-1} \times \mathbf{R}^{m-1} \times \mathbf{R} \rightarrow \mathbf{R}^{m-1}$ is a function for which we assume sufficient differentiability. We also assume $\partial F / \partial v^{\prime}$ to be singular with constant rank, (1.1) to be of index 1 over the whole interval of integration $\left[x_{0}, x_{\text {end }}\right]$, and the initial values to be consistent, i.e.,

$$
F\left(v_{0}, v_{0}^{\prime}, x_{0}\right)=0 \text {. }
$$

The index of a DAE is the number of times the algebraic part of the system has to be differentiated to obtain an ODE. The index 1 system (1.1) is supposed to be solvable in the sense that for each set of consistent initial values there exists a unique solution of the system. For more precise definitions of index and solvability, see [8].

According to Petzold [13], an $s$-stage Runge-Kutta method applied to (1.1) is defined by

$$
F\left(v_{n}+h \sum_{j=1}^{s} a_{i j} V_{j}^{\prime}, V_{i}^{\prime}, x_{n}+c_{i} h\right)=0, \quad i=1, \ldots, s,
$$

$$
v_{n+1}=v_{n}+h \sum_{i=1}^{s} b_{i} V_{i}^{\prime},
$$

Received May 27, 1988; revised April 14, 1989.

1980 Mathematics Subject Classification (1985 Revision). Primary 65L05. 
and the stage vectors $V_{i}$ are given by

$$
V_{i}=v_{n}+h \sum_{j=1}^{s} a_{i j} V_{j}^{\prime}, \quad i=1, \ldots, s .
$$

An $s$-stage Runge-Kutta method is described by its Butcher tableau

$$
\begin{array}{c|cccc}
c_{1} & a_{11} & a_{12} & \cdots & a_{1 s} \\
c_{2} & a_{21} & a_{22} & \cdots & a_{2 s} \\
\vdots & \vdots & \vdots & \ddots & \vdots \\
c_{s} & a_{s 1} & a_{s 2} & \cdots & a_{s s} \\
\hline & b_{1} & b_{2} & \cdots & b_{s}
\end{array}
$$

or by

$$
\begin{array}{l|l}
c & \mathscr{A} \\
\hline & b^{T},
\end{array}
$$

where $c_{i}=\sum_{j=1}^{s} a_{i j}, i=1, \ldots, s$. The matrix $\mathscr{A}$ has to be nonsingular, and we define

$$
\mathscr{D}=\left(d_{i j}\right)=\mathscr{A}^{-1}
$$

The local truncation error is given by

$$
d_{n+1}=\tilde{v}_{n+1}-v\left(x_{n}+h\right) \text {, }
$$

where $\tilde{v}_{n+1}$ is the solution of (1.3) when $v_{n}=v\left(x_{n}\right)$. The local order of the method is $k_{L}$ if

$$
d_{n+1}=\mathscr{O}\left(h^{k_{L}}\right)
$$

The global error is defined by

$$
e_{n}=v_{n}-v\left(x_{n}\right)
$$

and the order of the method is $k_{G}$ if

$$
e_{n}=\mathscr{O}\left(h^{k_{(i}}\right) \text {. }
$$

The stability constant $r$ is given by

$$
r=1-b^{T} \mathscr{A}^{-1} \varepsilon_{s}
$$

where $\varepsilon_{s}=[1, \ldots, 1]^{T} \in \mathbf{R}^{s}$.

Recently, the behavior of Runge-Kutta methods applied to differential-algebraic problems has received considerable attention. In [13], Petzold derived a complete set of order conditions for linear constant-coefficient index-1 equations, assuming that $|r|<1$. Under the same assumption, she also derived a sufficient set of order conditions for nonlinear problems (1.1) linear in $v^{\prime}$. Later, Burrage and Petzold [2] extended these results to also include the classes of methods with $|r|=1$. Kvaernø [9] derived a complete set of order conditions for the local truncation error for this class of problems by comparing the 
Taylor expansion of the exact and numerical solution of the equation. Roche $[14,15]$ derived general order conditions for Runge-Kutta methods applied to semiexplicit index-1 problems, using the theory of Butcher series and rooted trees. Very recently, this theory has been extended to the Hessenberg form index-2 DAE's by Hairer et al. [7].

The DAE (1.1) can (at least in theory) be transformed to an autonomous, partitioned system, by using the following arguments. The system can be written as an autonomous system with no loss of generality. This is done by adding the differential equation

$$
v_{m}^{\prime}=1, \quad v\left(x_{0}\right)=x_{0},
$$

with the solution $v_{m}(x)=x$, to the system. We then have

$$
F\left(v, v^{\prime}\right)=0
$$

with $v: \mathbf{R} \rightarrow \mathbf{R}^{m}$ and $F: \mathbf{R}^{m} \times \mathbf{R}^{m} \rightarrow \mathbf{R}^{m}$. Equation (1.5) can be split into a differential and an algebraic part. Gear [6] uses the following argument: Suppose that

$$
\operatorname{rank} F_{v^{\prime}}=r<m \text {. }
$$

Then there exists a nonsingular $r \times r$ submatrix of $F_{v^{\prime}}$. Suppose that the equations have been numbered so that rank $\partial f / \partial v^{\prime}=r$ over the whole interval of integration, where $f$ represents the first $r$ equations in $F$. Let $g$ be the last $m-r$ equations in $F$. Suppose that the variables are numbered such that

$$
\frac{\partial f}{\partial v^{\prime}}=\left[\frac{\partial f}{\partial v_{1}^{\prime}} \frac{\partial f}{\partial v_{2}^{\prime}}\right],
$$

where $v=\left[v_{1}^{T}, v_{2}^{T}\right]^{T}, v_{1} \in \mathbf{R}^{r}, v_{2} \in \mathbf{R}^{m-r}$, and $\partial f / \partial v_{1}^{\prime}$ is nonsingular. Then, by the implicit function theorem, $f=0$ can be solved for $v_{1}^{\prime}$, that is, $v_{1}^{\prime}=f_{1}\left(v_{1}, v_{2}, v_{2}^{\prime}\right)$. This can be substituted into the last $m-r$ equations to get an implicit relationship between $v_{1}$ and $v_{2}$. The vector $v_{2}^{\prime}$ cannot be involved, or we would be able to solve (1.5) for additional components of $v^{\prime}$, contrary to the assumption about the rank of $F_{v^{\prime}}$. Thus, (1.5) can be written as

$$
\begin{aligned}
f\left(v, v^{\prime}\right) & =0, \\
g(v) & =0 .
\end{aligned}
$$

The system (1.6) has index 1 if and only if

$$
\left[\begin{array}{l}
f_{v^{\prime}} \\
g_{v}
\end{array}\right]
$$

is nonsingular. In this paper, we are only concerned with solvable index-1 DAE's of the form (1.6). However, the results obtained are valid also for the more general form (1.1), as long as the rank of $\partial F / \partial v^{\prime}$ is constant over the whole interval of integration.

We have used a model equation for the derivation of the local order conditions. 
Theorem 1.1. The set of order conditions derived for the model equation

$$
\tilde{f}\left(y, z^{\prime}\right)=0, \quad z=\tilde{g}(y)
$$

is equivalent to the set of order conditions for the fully implicit problem (1.6).

Proof. The fully implicit index-1 DAE is given by (1.6). By the definition of the index, we know that

$$
\left[\begin{array}{l}
\partial f / \partial v^{\prime} \\
\partial g / \partial v
\end{array}\right]
$$

is nonsingular. Then, rank $g_{v}=m-r$, and there exists a nonsingular $(m-r) \times(m-r)$ submatrix of $g_{v}$. Suppose that the variables are now numbered such that $g_{v}$ can be written as

$$
\left[\frac{\partial g}{\partial y} \frac{\partial g}{\partial z}\right]
$$

where $v=\left[y^{T}, z^{T}\right]^{T}$, and $g_{z}$ is nonsingular. Then $g$ can be solved for $z$, and (1.6) can be written as

$$
f\left(y, z, y^{\prime}, z^{\prime}\right)=0, \quad z=\tilde{g}(y)
$$

or, by inserting the expression for $z$ into the differential equation, as

$$
f_{2}\left(y, y^{\prime}, z^{\prime}\right)=0, \quad z=\tilde{g}(y) .
$$

The numerical solution defined by (1.2) and (1.3), applied to (1.6), is given by

$$
f\left(V_{i}, V_{i}^{\prime}\right)=0, \quad g\left(V_{i}\right)=0 .
$$

The equation (1.8) is the same as (1.6) with $v$ replaced by $V_{i}$ and $v^{\prime}$ by $V_{i}^{\prime}$. Using the same arguments as above, (1.8) can be written as

$$
f_{2}\left(Y_{i}, Y_{i}^{\prime}, Z_{i}^{\prime}\right)=0, \quad Z_{i}=\tilde{g}\left(Y_{i}\right),
$$

where $V_{i}=\left[Y_{i}^{T}, Z_{i}^{T}\right]^{T}$. Note that the choice of $y$ and $z$ so that $g_{z}$ is nonsingular is not necessarily unique, neither will $g_{z}$ necessarily be constant over the whole interval of integration. But, at least in some neighborhood of the solution at $x_{n}, g_{z}$ will be nonsingular, and we assume $h$ to be small enough to keep $g_{z}$ nonsingular over the whole step. For the first step, (1.4) and (1.3) can be written as

$$
Y_{i}=y_{n}+h \sum_{j=1}^{s} a_{i j} Y_{j}^{\prime}, \quad Z_{i}=z_{n}+h \sum_{j=1}^{s} a_{i j} Z_{j}^{\prime}, \quad i=1, \ldots, s,
$$

and

$$
y_{n+1}=y_{n}+h \sum_{i=1}^{s} b_{i} Y_{i}^{\prime}, \quad z_{n+1}=z_{n}+h \sum_{i=1}^{s} b_{i} Z_{i}^{\prime} .
$$

By using (1.10) together with the algebraic part of (1.9) we have

$$
z_{n}+h \sum_{j=1}^{s} a_{i j} Z_{j}^{\prime}=\tilde{g}\left(y_{n}+h \sum_{j=1}^{s} a_{i j} Y_{j}^{\prime}\right),
$$


or, by solving for $Z_{i}^{\prime}$ and using the Taylor expansion around $\left(y_{n}, z_{n}\right)$,

$$
\begin{aligned}
Z_{i}^{\prime} & =\frac{1}{h} \sum_{j=1}^{s} d_{i j}\left(\tilde{g}\left(y_{n}+h \sum_{k=1}^{s} a_{j k} Y_{k}^{\prime}\right)-z_{n}\right) \\
& =\tilde{g}_{y} Y_{i}^{\prime}+\frac{h}{2} \sum_{j, k, l=1}^{s} d_{i j} a_{j k} a_{j l} \tilde{g}_{y y}\left(Y_{k}^{\prime}, Y_{l}^{\prime}\right)+\cdots
\end{aligned}
$$

Inserting this into the differential part of (1.9), we obtain

$$
f_{2}\left(Y_{i}, Y_{i}^{\prime}, Z_{i}^{\prime}\right)=f_{2}\left(Y_{i}, Y_{i}^{\prime}, \tilde{g}_{y} Y_{i}^{\prime}+\frac{h}{2} \sum_{j, k, l=1}^{s} d_{i j} a_{j k} a_{j l} \tilde{g}_{y y}\left(Y_{k}^{\prime}, Y_{l}^{\prime}\right)+\cdots\right)=0 \text {. }
$$

The term $Z_{i}^{\prime}$ involves $\tilde{g}_{y} Y_{i}^{\prime}$, so the term $Y_{i}^{\prime}$ in (1.9) will give no additional order conditions. We then have that the equation

$$
\tilde{f}\left(Y_{i}, Z_{i}^{\prime}\right)=0, \quad Z_{i}=\tilde{g}\left(Y_{i}\right), \quad i=1, \ldots, s,
$$

together with (1.11) and (1.10), will give all the necessary order conditions.

In $\S 2.1$ we develop a general scheme for the Taylor expansion of the exact solution of the model equation. In $\$ 2.2$ we give a complete set of order conditions for the local truncation error when a Runge-Kutta method is applied to the model equation. These order conditions take on a simple form, with the help of the "tree model" derived in $\S 2.1$. Convergence results are given in $\S 3$, while numerical experiments are described in $\S 4$.

\section{THE ORDER OF THE LOCAL TRUNCATION ERROR}

The aim of this section is to derive a set of necessary and sufficient order conditions for the local truncation error. In $\S 2.1$, we expand the solution of the model equation into a Taylor series. This series is expressed in terms of rooted trees. In $\S 2.2$, we derive the Taylor expansion of the numerical solution of the model equation. The coefficients of the Taylor series are obtained directly from the trees derived in $\S 2.1$. By comparing the Taylor series of the exact and numerical solution, the order conditions are obtained. The main result in this section is given in Theorem 2.2.

Some of the trees derived in $\S 2.1$ will correspond to identical order conditions. In $\S 2.3$, a reduced set of trees is introduced, so that each of the order conditions is given by one, and only one, tree. In Figure 2, all the order conditions up to order 4 are exhibited, together with their related trees.

2.1. Taylor expansion of the exact solution of a model equation. Consider the index-1 equation

$$
f\left(y, z^{\prime}\right)=0, \quad z=g(y)
$$

with consistent initial values $y\left(x_{0}\right)=y_{0}$ and $z\left(x_{0}\right)=z_{0}$, where $y: \mathbf{R} \rightarrow \mathbf{R}^{r}$, $z: \mathbf{R} \rightarrow \mathbf{R}^{m-r}, f: \mathbf{R}^{r} \times \mathbf{R}^{m-r} \rightarrow \mathbf{R}^{r}$, and $g: \mathbf{R}^{r} \rightarrow \mathbf{R}^{m-r}$. The functions $f$ and 
$g$ are assumed to be sufficiently differentiable. Repeated differentiation of the algebraic part of $(2.1)$ yields

$$
\begin{aligned}
z^{\prime} & =g_{y} y^{\prime} \\
z^{\prime \prime} & =g_{y y}\left(y^{\prime}, y^{\prime}\right)+g_{y} y^{\prime \prime} \\
z^{\prime \prime \prime} & =g_{y y y}\left(y^{\prime}, y^{\prime}, y^{\prime}\right)+g_{y y}\left(y^{\prime \prime}, y^{\prime}\right)+g_{y y}\left(y^{\prime}, y^{\prime \prime}\right)+g_{y y}\left(y^{\prime}, y^{\prime \prime}\right)+g_{y} y^{\prime \prime \prime}
\end{aligned}
$$

These expressions can be written in terms of trees as follows:
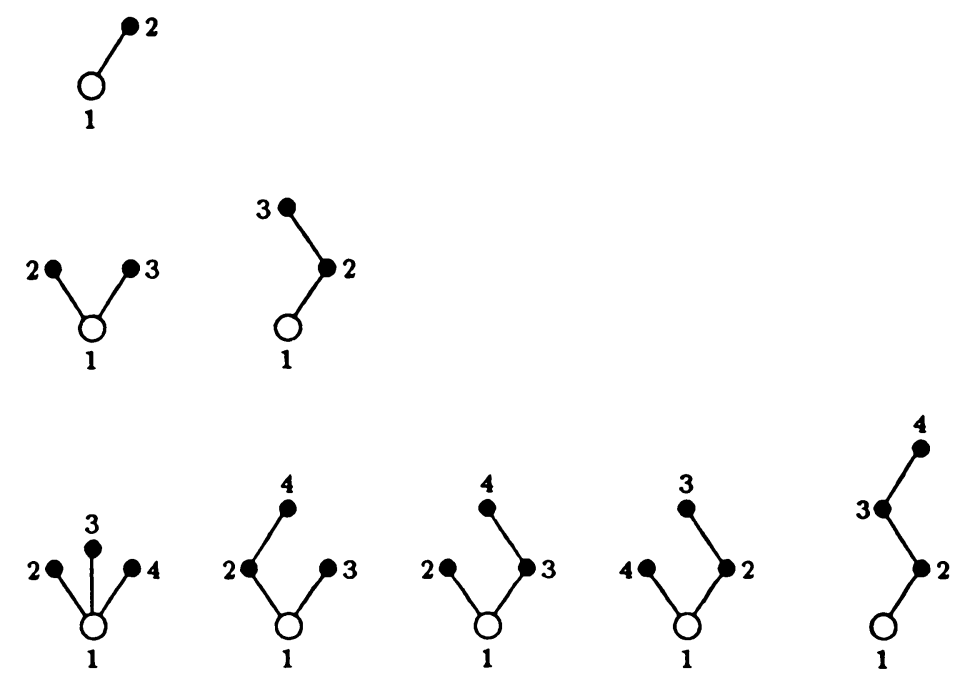

By inserting $z^{\prime}$ from (2.2) into the differential part of (2.1) we have

$$
f\left(y, g_{y} y^{\prime}\right)=0
$$

Since (2.1) is an index 1 equation, (2.3) can be solved for $y^{\prime}$, and $f_{z^{\prime}} g_{y}$ is nonsingular. Repeated differentiation of the differential part of (2.1) gives

$$
\begin{array}{r}
f_{y} y^{\prime}+f_{z^{\prime}} z^{\prime \prime}=0, \\
f_{y y}\left(y^{\prime}, y^{\prime}\right)+f_{y z^{\prime}}\left(y^{\prime}, z^{\prime \prime}\right)+f_{y} y^{\prime \prime}+f_{z^{\prime} y}\left(z^{\prime \prime}, y^{\prime}\right)+f_{z^{\prime} z^{\prime}}\left(z^{\prime \prime}, z^{\prime \prime}\right)+f_{z^{\prime}} z^{\prime \prime \prime}=0,
\end{array}
$$

By replacing the highest derivative of $z$ with the expression given in (2.2), we 
find

$$
\begin{aligned}
& y^{\prime}=y^{\prime} \\
& y^{\prime \prime}=\left(-f_{z^{\prime}} g_{y}\right)^{-1}\left(f_{z^{\prime}} g_{y y}\left(y^{\prime}, y^{\prime}\right)+f_{y} y^{\prime}\right) \\
& y^{\prime \prime \prime}=\left(-f_{z^{\prime}} g_{y}\right)^{-1}\left(f_{z^{\prime}} g_{y y y}\left(y^{\prime}, y^{\prime}, y^{\prime}\right)+f_{z^{\prime}} g_{y y}\left(y^{\prime \prime}, y^{\prime}\right)\right. \\
& \quad+f_{z^{\prime}} g_{y y}\left(y^{\prime}, y^{\prime \prime}\right)+f_{z^{\prime}} g_{y y}\left(y^{\prime}, y^{\prime \prime}\right) \\
& +f_{y y}\left(y^{\prime}, y^{\prime}\right)+f_{y z^{\prime}}\left(y^{\prime}, z^{\prime \prime}\right) \\
& \left.+f_{y} y^{\prime \prime}+f_{z^{\prime} y}\left(z^{\prime \prime}, y^{\prime}\right)+f_{z^{\prime} z^{\prime}}\left(z^{\prime \prime}, z^{\prime \prime}\right)\right)
\end{aligned}
$$

These expressions can also be written in terms of trees:

$:$
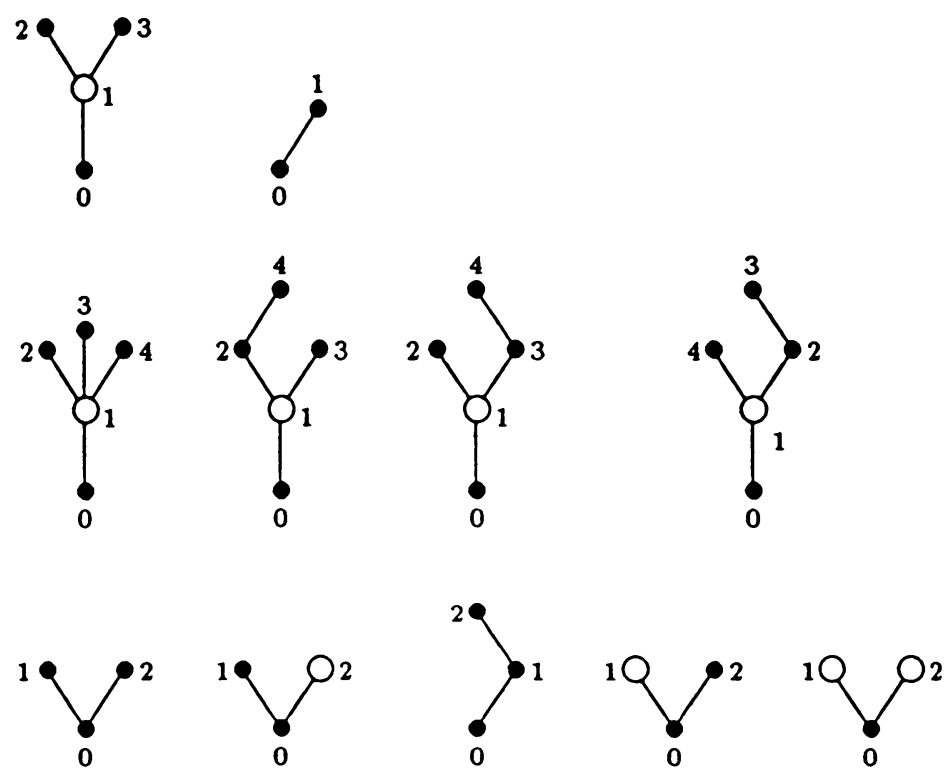

These graphs motivate us to introduce a set of special monotonically labelled trees, $t_{s}$, given by the following definitions.

Definition 2.1. The set of special trees, SDA1T, is the set of directed graphs, consisting of light and heavy vertices, with one single root, such that:

(1) $t_{s} \in \mathrm{SDA} \mathrm{T}_{y y}$ if the root is light, only the root has ramifications, and each of the branches consist of only light, or only heavy vertices. If the root has no ramification, then the tree consists of only light vertices.

(2) $t_{s} \in \mathrm{SDA}_{1 \mathrm{~T}}$ if the root is light without ramifications, but followed by a heavy vertex. The heavy vertex has at least two branches with no ramifications, and the branches all consist of only light vertices. 
(3) $t_{s} \in \mathrm{SDA}_{\mathrm{T}}$ if the root is heavy, only the root has ramifications, and the branches consist of only light vertices.

(4) $\mathrm{SDA}_{1 \mathrm{~T}}=\mathrm{SDA}_{y} \mathrm{~T}_{y y} \cup \mathrm{SDA} 1 \mathrm{~T}_{y z}$.

(5) $\mathrm{SDA} 1 \mathrm{~T}=\mathrm{SDA}_{1 \mathrm{~T}} \cup \mathrm{SDA}_{y} \mathrm{TT}_{z}$.

Let $\omega\left(t_{s}\right)$ be the number of vertices in a tree.

Definition 2.2. Let $t_{s} \in \mathrm{SDA} 1 \mathrm{~T}$. We say that $t_{s}$ is monotonically labelled if every vertex is associated with an integer $i$ satisfying

$$
\begin{gathered}
0 \leq i \leq \omega\left(t_{s}\right)-1 \quad \text { if } t_{s} \in \mathrm{SDA}_{1 \mathrm{~T}}, \\
1 \leq i \leq \omega\left(t_{s}\right) \quad \text { if } t_{s} \in \mathrm{SDA}_{\mathrm{ST}},
\end{gathered}
$$

and if, following each branch of $t_{s}$, the labels are monotonically increasing.

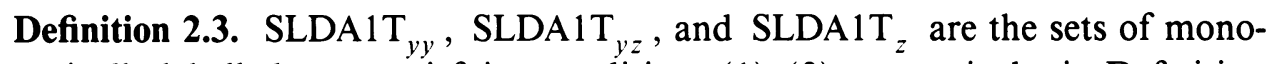
tonically labelled trees satisfying conditions (1)-(3), respectively, in Definition 2.1 , and

$$
\begin{gathered}
\mathrm{SLDA}_{1 \mathrm{~T}}=\mathrm{SLDA}_{y} \mathrm{TT}_{y y} \cup \mathrm{SLDA}_{1 \mathrm{~T}}, \\
\mathrm{SLDA} 1 \mathrm{~T}=\mathrm{SLDA}_{y z} \cup \mathrm{SLDA}_{y} \mathrm{ST}_{z} .
\end{gathered}
$$

The set of trees, SLDA1T, corresponds to the SLDAT-trees defined by Roche [14]. The differences between the two sets of trees derives from the fact that the trees of Roche are constructed for a semiexplicit index 1 equation, while the trees used in this paper are constructed for the model equation (2.1). Also, in the rest of this paper, we will use similar notations as used by Roche. To distinguish between the two kind of trees, we use the notation DA1T-trees in place of the DAT-trees used by Roche.

Let $\omega_{m}\left(t_{s}\right)$ be the number of light vertices, and let $\omega_{f}\left(t_{s}\right)$ be the number of heavy vertices in $t_{s} \in$ SLDA $1 T$.

Definition 2.4. The order $\rho\left(t_{s}\right)$ of a tree $t_{s} \in \mathrm{SLDA} 1 \mathrm{~T}$ is defined by

(1) $\rho\left(t_{s}\right)=\omega_{m}\left(t_{s}\right)+\omega_{f}\left(t_{s}\right)$ if $t_{s} \in \mathrm{SLDA}_{1 \mathrm{~T}}$,

(2) $\rho\left(t_{s}\right)=\omega_{m}\left(t_{s}\right)-1$ if $t_{s} \in \operatorname{SLDA} 1 \mathrm{~T}_{y z}$,

(3) $\rho\left(t_{s}\right)=\omega_{m}\left(t_{s}\right)$ if $t_{s} \in \mathrm{SLDA}_{1 \mathrm{~T}}$.

Then $t_{s}$ is the tree representation for one of the terms in $y^{\left(\rho\left(t_{s}\right)\right)}$ if $t_{s} \in$ $\mathrm{SLDA}_{1 \mathrm{~T}}$, and one of the terms in $z^{\left(\rho\left(t_{s}\right)\right)}$ if $t_{s} \in \mathrm{SLDA}_{\mathrm{T}}$. Let

$$
\tau_{y}=\bullet \quad \tau_{z}=\sigma
$$

Definition 2.5. For every tree $t_{s} \in \mathrm{SDA}_{\mathrm{ST}}$, we define a function $F_{s}\left(t_{s}\right): \mathbf{R}^{r} \times$ $\mathbf{R}^{m-r} \rightarrow \mathbf{R}^{r}$, and for every tree $u_{s} \in{\operatorname{SDA} 1 T_{z}}_{z}$ we define a function $G_{s}\left(u_{s}\right): \mathbf{R}^{r} \times$ $\mathbf{R}^{m-r} \rightarrow \mathbf{R}^{m-r}$, by

(1) $F_{s}\left(\tau_{y}\right)(y, z)=y^{\prime}$ and $G_{s}\left(\tau_{z}\right)(y, z)=g_{y} y^{\prime}=z^{\prime}$, 
(2) $F_{s}\left(t_{s}\right)(y, z)=\left(-f_{z^{\prime}} g_{y}\right)^{-1} f_{k y l z^{\prime}}\left(y^{\left(p_{1}\right)}, \ldots, y^{\left(p_{k}\right)}, z^{\left(q_{1}\right)}, \ldots, z^{\left(q_{l}\right)}\right)$ if $t_{s} \in$ $\mathrm{SDA}_{1 \mathrm{~T}_{y y}}$

(3) $F_{s}\left(t_{s}\right)(y, z)=\left(-f_{z^{\prime}} g_{y}\right)^{-1} f_{z^{\prime}} g_{k y}\left(y^{\left(p_{1}\right)}, \ldots, y^{\left(p_{k}\right)}\right)$ if $t_{s} \in \mathrm{SDA}_{1 \mathrm{~T}}$,

(4) $G_{s}\left(u_{s}\right)(y, z)=g_{k y}\left(y^{\left(p_{1}\right)}, \ldots, y^{\left(p_{k}\right)}\right)$ if $u_{s} \in \operatorname{SDA} 1 \mathrm{~T}_{z}$,

where the tree $t_{s}$ or $u_{s}$ has $k$ light branches with respectively $p_{1}, \ldots, p_{k}$ vertices, and $l$ heavy branches with respectively $q_{1}-1, \ldots, q_{l}-1$ vertices.

Example 2.1. The tree

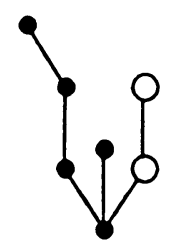

corresponds to $\left(-f_{z^{\prime}} g_{y}\right)^{-1} f_{y y z^{\prime}}\left(y^{\prime \prime \prime}, y^{\prime}, z^{\prime \prime \prime}\right)$.

There is a one-to-one correspondence between the trees $t_{s} \in \mathrm{SLDA}_{1 \mathrm{~T}}$, $\rho\left(t_{s}\right)=p$, and the terms of $y^{(p)}$, and between the trees $u_{s} \in \operatorname{SLDA} \mathrm{ST}_{z}$, $\rho\left(u_{s}\right)=p$, and the terms in $z^{(p)}$. The following arguments will show this. The trees corresponding to the terms in $y^{\prime}, y^{\prime \prime}, y^{\prime \prime \prime}, z^{\prime}, z^{\prime \prime}$, and $z^{\prime \prime \prime}$ are already given. Suppose that all the trees corresponding to $y^{\prime}, \ldots, y^{(p)}$ and $z^{\prime}, \ldots, z^{(p)}$ are given. Let $u_{s} \in \mathrm{SLDA} 1 \mathrm{~T}_{z}$, with $\rho\left(t_{s}\right)=p$. Attach a light vertex once to each of the terminal vertices of the tree, and once to the root. Associate with the new vertex the number $\omega\left(u_{s}\right)+1$. Do this with all the trees $u_{s} \in \operatorname{SLDA}_{z}$, $\rho\left(u_{s}\right)=p$. Now we have the set of trees corresponding to $z^{(p+1)}$. To find the trees corresponding to $y^{(p+1)}$, we first have to differentiate $f^{(p)}$. Let $T_{p}$ be the monotonically labelled tree with a light root followed by one single branch with $p$ heavy vertices. This tree corresponds to the term $\left(-f_{z^{\prime}} g_{y}\right)^{-1} f_{z^{\prime}} z^{(p)}$. The derivative $f^{(p)}$ is composed of terms obtained by premultiplying the derivatives corresponding to the trees $t_{s} \in \mathrm{SLDA}_{1 \mathrm{~T}} \cup T_{p}$ of order $\rho\left(t_{s}\right)=p$ with $\left(-f_{z^{\prime}} g_{y}\right)$. To find the trees corresponding to $\left(-f_{z^{\prime}} g_{y}\right)^{-1} f^{(p+1)}$, attach a light vertex once to each terminal light vertex and once to the root. This corresponds to differentiation of $\left(-f_{z^{\prime}} g_{y}\right)^{-1} f^{(p)}$ with respect to $y$. Then attach a heavy vertex once to each heavy terminal vertex, and once to the root. This corresponds to differentiating $\left(-f_{z^{\prime}} g_{y}\right)^{-1} f^{(p)}$ with respect to $z^{\prime}$. Associate with the new vertex the integer $\omega\left(t_{s}\right)+1$. We now have all the trees in SLDA1T with $\rho\left(t_{s}\right)=p+1$, and the tree $T_{p+1}$ corresponding to $\left(-f_{z^{\prime}} g_{y}\right)^{-1} f_{z^{\prime}} z^{(p+1)}$. Replace the heavy branch in $T_{p+1}$ with the trees corresponding to $z^{(p+1)}$. This will give the trees $t_{s} \in \mathrm{SLDAlT}_{y z} \cup U_{p+1}$ with $\rho\left(t_{s}\right)=p+1$. The tree $U_{p+1}$ consists of a light root followed by one branch with one heavy vertex, followed by $p+1$ light vertices. This tree corresponds to $-y^{(p+1)}$, for which the equation $\left(-f_{z^{\prime}} g_{y}\right)^{-1} f^{(p+1)}=0$ is solved. 
The number of ways to label a tree $t_{s} \in \mathrm{SDA} 1 \mathrm{~T}$ is the number of times the corresponding derivative appears in the Taylor expansion of the exact solution. We call this number $\beta\left(t_{s}\right)$. We can now state the following lemma.

Lemma 2.1. For the exact solution of (2.1) we have

$$
\begin{aligned}
& y^{(p)}=\sum_{\substack{t_{s} \in \mathrm{SLDA}_{s} \mathrm{~L}_{y} \\
\rho\left(t_{s}\right)=p}} F_{s}\left(t_{s}\right)=\sum_{\substack{t_{s} \in \mathrm{SDA}^{\prime} \mathrm{T}_{y} \\
\rho\left(t_{s}\right)=p}} \beta\left(t_{s}\right) F_{s}\left(t_{s}\right), \\
& z^{(p)}=\sum_{\substack{u_{s} \in \mathrm{SLDA} 1 \mathrm{~T}=\\
\rho\left(u_{s}\right)=p}} G_{s}\left(u_{s}\right)=\sum_{\substack{u_{s} \in \mathrm{SDA} 1 \mathrm{~T} \\
\rho\left(u_{s}\right)=p}} \beta\left(u_{s}\right) G_{s}\left(u_{s}\right) .
\end{aligned}
$$

We now know how to express $y^{(p)}$ and $z^{(p)}$ in terms of partial derivatives of $f$ and $g$, and of lower derivatives of $y$ and $z$. What we want is to express $y^{(p)}$ and $z^{(p)}$ in terms of partial derivatives of $f$ and $g$, and of $y^{\prime}$. Such an expression is already given for $y^{\prime \prime}$ in (2.4). By inserting this into the expression for $z^{\prime \prime}$ in (2.2) we obtain

$$
z^{\prime \prime}=g_{y y}\left(y^{\prime}, y^{\prime}\right)+g_{y}\left(-f_{z^{\prime}} g_{y}\right)^{-1} f_{y} y^{\prime}+g_{y}\left(-f_{z^{\prime}} g_{y}\right)^{-1} f_{z^{\prime}} g_{y y}\left(y^{\prime}, y^{\prime}\right) .
$$

The expressions for $y^{\prime \prime}$ and $z^{\prime \prime}$ can be inserted into the expression for $y^{\prime \prime \prime}$, and then for $z^{\prime \prime \prime}$, etc. We now find a new set of trees corresponding to these expressions. This set is defined as follows.

Definition 2.6. We denote by DA1T, DA1T , $_{y}$ and $\mathrm{DA}_{\mathrm{T}} \mathrm{T}_{z}$ the set of trees defined recursively by

1. $\tau_{y} \in \mathrm{DAlT}_{y}$ and $\tau_{z} \in \mathrm{DAlT}_{z y}$.

2. (a) If $t_{1}, \ldots, t_{k} \in \mathrm{DA}_{1} \mathrm{~T}_{y}$ and $k>1$, then $\left[t_{1}, \ldots, t_{k}\right]_{z} \in \mathrm{DA} 1 \mathrm{~T}_{z z}$.

(b) If $t_{1}, \ldots, t_{k} \in \mathrm{DA}_{1} \mathrm{~T}_{y}, u_{1}, \ldots, u_{l} \in \mathrm{DA}_{1} \mathrm{~T}_{z} \backslash\left\{\tau_{z}\right\}, k>0$ or $k=0$, and $l>1$, then $\left[t_{1}, \ldots t_{k}, u_{1}, \ldots, u_{l}\right]_{y} \in \mathrm{DA} \mathrm{DT}_{y y}$.

(c) If $u \in \mathrm{DA}_{1 \mathrm{~T}} z$ then $[u]_{y} \in \mathrm{DA}_{1 \mathrm{~T}} \mathrm{D}_{y z}$.

(d) If $t \in \mathrm{DA}_{1 \mathrm{~T}}$ then $[t]_{z} \in \mathrm{DAlT}_{z y}$.

3. $\mathrm{DA}_{1} \mathrm{~T}_{y}=\mathrm{DA}_{1 \mathrm{~T}} \mathrm{D}_{y} \cup \mathrm{DA}_{1} \mathrm{~T}_{y z}, \mathrm{DA}_{1} \mathrm{~T}_{z}=\mathrm{DA} \mathrm{T}_{z y} \cup \mathrm{DA} 1 \mathrm{~T}_{z z}$.

4. $\mathrm{DA} 1 \mathrm{~T}=\mathrm{DA}_{1} \mathrm{~T}_{y} \cup \mathrm{DA} 1 \mathrm{~T}_{z}$.

Here, $t=\left[t_{1}, \ldots, t_{k}, u_{1}, \ldots, u_{l}\right]_{v}$ is the tree obtained by connecting the roots of $t_{1}, \ldots, t_{k}, u_{1}, \ldots, u_{l}$ by $k+l$ arcs to a new light vertex which becomes the new root of $t$. Similarly, $u=\left[t_{1}, \ldots, t_{k}\right]_{z}$ is the tree obtained in the same manner, but with a new heavy root.

Definition 2.7. The order $\rho(t)$ of a tree $t \in \mathrm{DA} 1 \mathrm{~T}$ is given by

(1) $\rho(t)=\omega_{m}(t)-\omega_{f}(t)$ if $t \in \mathrm{DA}_{1} \mathrm{~T}_{y}$,

(2) $\rho(t)=\omega_{m}(t)-\omega_{f}(t)+1$ if $t \in \mathrm{DA} 1 \mathrm{~T}_{z}$,

where $\omega_{m}(t)$ and $\omega_{f}(t)$ are the number of light, resp. heavy, vertices in the tree. 
There is a one-to-one correspondence between these trees and the terms appearing in the Taylor expansion of the exact solution. This correspondence is given in the following definition.

Definition 2.8. For every tree $t \in \mathrm{DAlT}_{y}$ we define a function $F(t)(y, z): \mathbf{R}^{r} \times$ $\mathbf{R}^{m-r} \rightarrow \mathbf{R}^{r}$, and for every tree $u \in \mathrm{DA}_{1} \mathrm{~T}_{z}$ we define a function $G(u)(y, z)$ : $\mathbf{R}^{r} \times \mathbf{R}^{m-r} \rightarrow \mathbf{R}^{m-r}$ recursively by:

(1) $F\left(\tau_{y}\right)(y, z)=y^{\prime}, G\left(\tau_{z}\right)(y, z)=z^{\prime}=g_{y} y^{\prime}$,

(2) $F(t)(y, z)=\left(-f_{z^{\prime}} g_{y}\right)^{-1} f_{k y l z^{\prime}}\left(F\left(t_{1}\right), \ldots, F\left(t_{k}\right), G\left(u_{1}\right), \ldots, G\left(u_{l}\right)\right)$ if $t=\left[t_{1}, \ldots, t_{k}, u_{1}, \ldots, u_{l}\right]_{y}$,

(3) $G(u)(y, z)=g_{k y}\left(F\left(t_{1}\right), \ldots, F\left(t_{k}\right)\right)$ if $u=\left[t_{1}, \ldots, t_{k}\right]_{z}$,

where $t_{1}, \ldots, t_{k} \in \mathrm{DA} 1 \mathrm{~T}_{y}$ and $u_{1}, \ldots, u_{l} \in \mathrm{DA}_{\mathrm{T}}$. The expressions $F(t)(y, z)$ and $G(u)(y, z)$ are called the elementary differentials associated with the tree $t$, respectively $u$.

$\begin{array}{lll}y^{\prime} & \rho(t)=1 & \tau_{y} \\ g_{y} y^{\prime} & \rho(u)=1 & \tau_{z} \\ \left(-f_{z^{\prime}} g_{y}\right)^{-1} f_{y} y^{\prime} & \rho(t)=2 & {\left[\tau_{y}\right]_{y}} \\ \left(-f_{z^{\prime}} g_{y}\right)^{-1} f_{z^{\prime}} g_{y y}\left(y^{\prime}, y^{\prime}\right) & \rho(t)=2 & {\left[\left[\tau_{y}, \tau_{y}\right]_{z}\right]_{y}} \\ g_{y y}\left(y^{\prime}, y^{\prime}\right) & \rho(u)=2 \\ g_{y}\left(-f_{z^{\prime}} g_{y}\right)^{-1} f_{y} y^{\prime} & {\left[\tau_{y}, \tau_{y}\right]_{z}} \\ g_{y}\left(-f_{z^{\prime}} g_{y}\right)^{-1} f_{z^{\prime}} g_{y y}\left(y^{\prime}, y^{\prime}\right) & {[(u)=2}\end{array}$

FIGURE 1. Elementary differentials and corresponding trees 
Figure 1 shows the elementary differentials and the corresponding trees for $y^{\prime}, z^{\prime}, y^{\prime \prime}$, and $z^{\prime \prime}$.

There exists a relation between the trees of SDA1T and those of DA1T. Let $t_{s} \in \mathrm{SDA}_{1 \mathrm{~T}}$ and

$$
F_{s}\left(t_{s}\right)=\left(-f_{z^{\prime}} g_{y}\right)^{-1} f_{k y l z^{\prime}}\left(y^{\left(p_{1}\right)}, \ldots, y^{\left(p_{k}\right)}, z^{\left(q_{1}\right)}, \ldots, z^{\left(q_{l}\right)}\right)
$$

with $\rho\left(t_{s}\right)=p$. Suppose that all the trees $t \in$ DA1T, $\rho(t) \leq p$, are given. Replace each branch with $p_{i}$ light vertices once with each of the trees $t \in$ DA1T $_{y}, \rho(t)=p_{i}$, and each of the branches with $q_{j}-1$ heavy vertices once with each of the trees $u \in \mathrm{DA}_{1 \mathrm{~T}}, \rho(u)=q_{j}$. Then we have the set of trees $t \in \mathrm{DA} 1 \mathrm{~T}, \rho(t)=p$. See Example 2.2.

Example 2.2. Let $t_{s} \in \mathrm{SDA}_{1 \mathrm{~T}}, \rho\left(t_{s}\right)=4$, be the tree

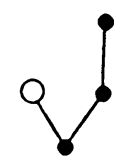

with corresponding derivative $\left(-f_{z^{\prime}} g_{y}\right)^{-1} f_{z^{\prime} y}\left(z^{\prime \prime}, y^{\prime \prime}\right)$. Replace the light branch with each of the trees corresponding to $y^{\prime \prime}$, that is all the trees given in Figure 1 with a light root and $\rho(t)=2$. We then obtain all the trees of DA1T corresponding to $t_{s}$ :

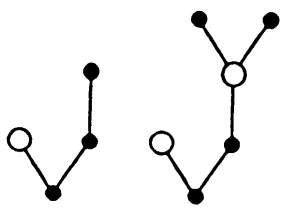

Then replace the heavy branch with each of the trees corresponding to $z^{\prime \prime}$, that is all the trees given in Figure 1 with a heavy root and $\rho(u)=2$ :
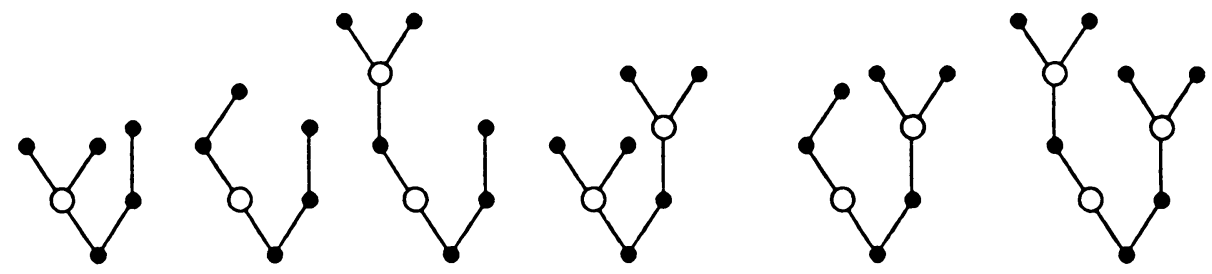

Similar transformations can be carried out with all the trees in SDA1T. For each $t_{s} \in \mathrm{SDA} 1 \mathrm{~T}$ there is a corresponding set of trees $t \in \mathrm{DA} 1 \mathrm{~T}$. Let $t \in$ DA1T be one of the trees obtained from a tree $t_{s} \in$ SDA1T as described above. Then we call $t_{s}$ the special tree corresponding to $t$, and denote it by $S(t)$. This is illustrated in the following example. 
Example 2.3. Let $t_{1} \in \mathrm{SDA}_{1 \mathrm{~T}} \mathrm{~T}_{y y}$ and $t_{2} \in \mathrm{SDA}_{y z}$. The corresponding special trees are given by
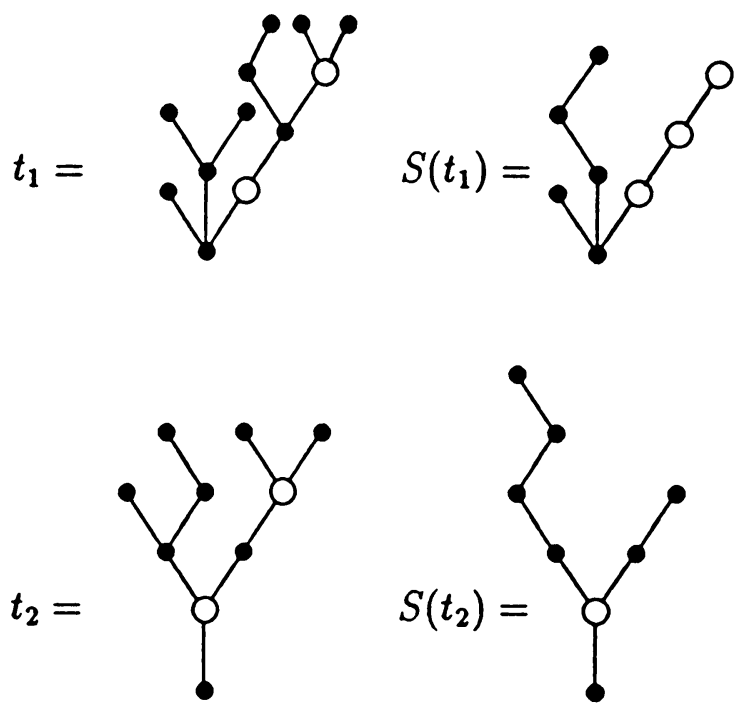

Let $\alpha(t)$ be the number of times the elementary differential corresponding to $t \in$ DA1T appears in the exact solution of the model equation (2.1). We can then state the following result.

Theorem 2.1. For the exact solution of (2.1) we have

$$
\begin{aligned}
y^{(p)}\left(x_{0}\right) & =\sum_{\substack{t \in \mathrm{DA}^{\prime} \mathrm{T}_{y} \\
\rho(t)=p}} \alpha(t) F(t)\left(y_{0}, z_{0}\right), \\
z^{(p)}\left(x_{0}\right) & =\sum_{\substack{u \in \mathrm{DA}_{1 \mathrm{~T}} \\
\rho(u)=p}} \alpha(u) G(u)\left(y_{0}, z_{0}\right)
\end{aligned}
$$

and

$$
\begin{aligned}
& y\left(x_{0}+h\right)=y_{0}+\sum_{t \in \mathrm{DAlT}_{y}} \alpha(t) F(t)\left(y_{0}, z_{0}\right) \frac{h^{\rho(t)}}{\rho(t) !}, \\
& z\left(x_{0}+h\right)=z_{0}+\sum_{u \in \mathrm{DAlT}_{z}} \alpha(u) G(u)\left(y_{0}, z_{0}\right) \frac{h^{\rho(u)}}{\rho(u) !} .
\end{aligned}
$$

Lemma 2.2. $\alpha(t)$ is given recursively by

(1) $\alpha\left(\tau_{y}\right)=\alpha\left(\tau_{z}\right)=1$.

(2) If $t=\left[t_{1}, \ldots, t_{k}, u_{1}, \ldots, u_{l}\right]_{y}$, then $\alpha(t)=\beta(S(t)) \alpha\left(t_{1}\right) \cdots \alpha\left(t_{k}\right) \alpha\left(u_{1}\right)$ $\cdots \alpha\left(u_{l}\right)$, and if $u=\left[t_{1}, \ldots, t_{k}\right]_{z}$, then $\alpha(u)=\beta(S(u)) \alpha\left(t_{1}\right) \cdots \alpha\left(t_{k}\right)$. 
Proof. From Lemma 2.1, Definitions 2.5 and 2.8, and Theorem 2.1 we have

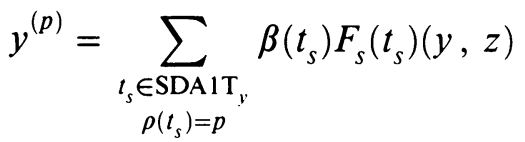

$$
\begin{aligned}
& =\sum_{\substack{t_{s} \in \operatorname{SDA}^{\prime} \mathrm{T}_{y} \\
\rho\left(t_{s}\right)=p}} \beta\left(t_{s}\right)\left(-f_{z^{\prime}} g_{y}\right)^{-1} f_{k y l z^{\prime}}\left(y^{\left(p_{1}\right)}, \ldots, y^{\left(p_{k}\right)}, z^{\left(q_{1}\right)}, \ldots, z^{\left(q_{l}\right)}\right) \\
& =\sum_{\substack{t_{s} \in \mathrm{SDAT}_{j} \\
\rho\left(t_{s}\right)=p}} \beta\left(t_{s}\right)\left(-f_{z^{\prime}} g_{y}\right)^{-1} f_{k y l z^{\prime}} \\
& \cdot\left(\sum_{\substack{t_{1} \in \mathrm{DA}_{1 \mathrm{~T}} \\
\rho\left(t_{1}\right)=p_{1}}} \alpha\left(t_{1}\right) F\left(t_{1}\right)(y, z), \ldots, \sum_{\substack{u_{l} \in \mathrm{DA} 1 \mathrm{~T} \\
\rho\left(u_{l}\right)=q_{l}}} \alpha\left(u_{l}\right) G\left(u_{l}\right)(y, z)\right) .
\end{aligned}
$$

Let $S(t) \in \operatorname{SDAlT}_{y}, t=\left[t_{1}, \ldots, t_{k}, u_{1}, \ldots, u_{l}\right]_{y}$, and $\rho(t)=p$ be one of the trees in (2.7). The value of $\alpha(t)$ is given directly by comparing (2.7) with (2.5). A similar procedure can be used to prove the lemma for $\alpha(u)$.

2.2. Taylor expansion of the numerical solution of the model equation. To be able to find the order conditions for Runge-Kutta methods applied to the model equation (2.1), we have to find the Taylor expansion of the numerical solution. To do this, we introduce the concept of DA1-series. Similar series are given by Roche [14] for semiexplicit index-1 problems.

Definition 2.9. Let a: $\mathrm{DA}_{1} \mathrm{~T}_{y} \rightarrow \mathbf{R}$ and $\mathbf{b}: \mathrm{DA}_{1} \mathrm{~T}_{z} \rightarrow \mathbf{R}$ be any mappings. The series

$$
\begin{aligned}
& \operatorname{DA}_{y}\left(\mathbf{a}, y_{0}, z_{0}\right)=y_{0}+\sum_{t \in \mathrm{DAlT}_{y}} \mathbf{a}(t) \alpha(t) F(t) \frac{h^{\rho(t)}}{\rho(t) !}, \\
& \mathrm{DA}_{z}\left(\mathbf{b}, y_{0}, z_{0}\right)=z_{0}+\sum_{u \in \mathrm{DAlT}} \mathbf{b}(u) \alpha(u) G(u) \frac{h^{\rho(u)}}{\rho(u) !}
\end{aligned}
$$

are called $\mathrm{DA} 1_{y}$, respectively $\mathrm{DA} 1_{z}$, series.

A Runge-Kutta method applied to (2.1) is given by

$$
\begin{gathered}
f\left(y_{0}+h \sum_{j=1}^{s} a_{i j} Y_{j}^{\prime}, Z_{i}^{\prime}\right)=0, \quad i=1, \ldots, s, \\
z_{0}+h \sum_{j=1}^{s} a_{i j} Z_{j}^{\prime}=g\left(y_{0}+h \sum_{j=1}^{s} a_{i j} Y_{j}^{\prime}\right), \\
y_{1}=y_{0}+h \sum_{i=1}^{s} b_{i} Y_{i}^{\prime}, \quad z_{1}=z_{0}+h \sum_{i=1}^{s} b_{i} Z_{i}^{\prime} .
\end{gathered}
$$


The stage values are given by

$$
\begin{aligned}
& Y_{i}=y_{0}+h \sum_{j=1}^{s} a_{i j} Y_{j}^{\prime}, \\
& Z_{i}=z_{0}+h \sum_{j=1}^{s} a_{i j} Z_{j}^{\prime},
\end{aligned}
$$

Now $Y_{i}, y_{1}$, respectively $Z_{i}$ and $z_{1}, i=1, \ldots, s$, can be written as $\mathrm{DA}_{y}$, respectively $\mathrm{DA}_{z}$, series as follows:

$$
\begin{aligned}
Y_{i}\left(x_{0}+h\right) & =y_{0}+\sum_{t \in \mathrm{DA} I \mathrm{~T}_{y}} \mathbf{v}_{i}(t) \alpha(t) F(t) \frac{h^{\rho(t)}}{\rho(t) !}, \\
Z_{i}\left(x_{0}+h\right)=z_{0}+\sum_{u \in \mathrm{DA} I \mathrm{~T}_{z}} \mathbf{w}_{i}(u) \alpha(u) G(u) \frac{h^{\rho(u)}}{\rho(u) !}, & i \\
y_{1}\left(x_{0}+h\right) & =y_{0}+\sum_{t \in \mathrm{DA} 1 \mathrm{~T}_{y}} \mathbf{y}_{1}(t) \alpha(t) F(t) \frac{h^{\rho(t)}}{\rho(t) !}, \\
z_{1}\left(x_{0}+h\right) & =z_{0}+\sum_{u \in \mathrm{DAlT}_{z}} \mathbf{z}_{1}(u) \alpha(u) G(u) \frac{h^{\rho(u)}}{\rho(u) !} .
\end{aligned}
$$

The stage derivatives are written as

$$
\begin{aligned}
& Y_{i}^{\prime}\left(x_{0}+h\right)=\sum_{t \in \mathrm{DA} I \mathrm{~T}_{y}} \mathbf{l}_{i}(t) \alpha(t) F(t) \frac{h^{\rho(t)-1}}{\rho(t) !}, \quad i=1, \ldots, s . \\
& Z_{i}^{\prime}\left(x_{0}+h\right)=\sum_{u \in \mathrm{DA} I \mathrm{~T}_{z}} \mathbf{k}_{i}(u) \alpha(u) G(u) \frac{h^{\rho(u)-1}}{\rho(u) !},
\end{aligned}
$$

By inserting (2.13) into (2.10) we get

$$
\begin{aligned}
& Y_{i}\left(x_{0}+h\right)=y_{0}+h \sum_{j=1}^{s} a_{i j} Y_{j}^{\prime}=y_{0}+\sum_{t \in \mathrm{DA} I \mathrm{~T}_{y}}\left(\sum_{j=1}^{s} a_{i j} \mathbf{l}_{j}(t)\right) \alpha(t) F(t) \frac{h^{\rho(t)}}{\rho(t) !}, \\
& Z_{i}\left(x_{0}+h\right)=z_{0}+h \sum_{j=1}^{s} a_{i j} Z_{j}^{\prime}=z_{0}+\sum_{u \in \mathrm{DA} I \mathrm{~T}_{z}}\left(\sum_{j=1}^{s} a_{i j} \mathbf{k}_{j}(u)\right) \alpha(u) G(u) \frac{h^{\rho(u)}}{\rho(u) !} .
\end{aligned}
$$

Comparing this with (2.11), we have

$$
\mathbf{v}_{i}(t)=\sum_{j=1}^{s} a_{i j} \mathbf{l}_{j}(t), \quad \mathbf{w}_{i}(t)=\sum_{j=1}^{s} a_{i j} \mathbf{k}_{j}(u) .
$$

Similarly, by inserting (2.13) into (2.9) and comparing with (2.12), we have

$$
\mathbf{y}_{1}(t)=\sum_{i=1}^{s} b_{i} \mathbf{l}_{i}(t), \quad \mathbf{z}_{1}(u)=\sum_{i=1}^{s} b_{i} \mathbf{k}_{i}(u) .
$$


The Taylor expansion of the numerical solution can be written as

$$
\begin{array}{clrl}
Y_{i}^{\prime}=\sum_{p=1}^{\infty} \widetilde{Y}_{i}^{(p)} \frac{h^{p-1}}{p !}, & Z_{i}^{\prime}=\sum_{p=1}^{\infty} \widetilde{Z}_{i}^{(p)} \frac{h^{p-1}}{p !}, \\
Y_{i}=y_{0}+\sum_{p=1}^{\infty} \bar{Y}_{i}^{(p)} \frac{h^{p}}{p !}, & Z_{i}=z_{0}+\sum_{p=1}^{\infty} \bar{Z}_{i}^{(p)} \frac{h^{p}}{p !}, \\
y_{1}=y_{0}+\sum_{p=1}^{\infty} \tilde{y}^{(p)} \frac{h^{p}}{p !}, & z_{1}=z_{0}+\sum_{p=1}^{\infty} \tilde{z}^{(p)} \frac{h^{p}}{p !} .
\end{array}
$$

Comparing (2.13) with (2.16), (2.11) with (2.17), and (2.12) with (2.18), we have

$$
\begin{aligned}
& \tilde{Y}_{i}^{(p)}=\sum_{\substack{t \in \mathrm{DA}^{\prime} \mathrm{T}_{y} \\
\rho(t)=p}} \mathbf{l}_{i}(t) \alpha(t) F(t), \quad \widetilde{Z}_{i}^{(p)}=\sum_{\substack{u \in \mathrm{DA}^{\prime} \mathrm{T}_{i} \\
\rho(u)=p}} \mathbf{k}_{i}(u) \alpha(u) G(u), \\
& \text { (2.20) } \quad \bar{Y}_{i}^{(p)}=\sum_{\substack{t \in \mathrm{DA}^{\prime} \mathrm{T}_{y} \\
\rho(t)=p}} \mathbf{v}_{i}(t) \alpha(t) F(t), \quad \bar{Z}_{i}^{(p)}=\sum_{\substack{u \in \mathrm{DAlT}^{\rho}=\\
\rho(u)=p}} \mathbf{w}_{i}(u) \alpha(u) G(u), \\
& \text { (2.21) } \quad \tilde{y}^{(p)}=\sum_{\substack{t \in \mathrm{DA}^{\prime} \mathrm{T}_{y} \\
\rho(t)=p}} \mathbf{y}_{1}(t) \alpha(t) F(t), \quad \tilde{z}^{(p)}=\sum_{\substack{u \in \mathrm{DA} \mid \mathrm{T} \\
\rho(u)=p}} \mathbf{z}_{1}(u) \alpha(u) G(u) .
\end{aligned}
$$

We use the notation $\tilde{Y}_{i}^{\left(p_{k}\right)}\left(t_{k}\right)$ for the term $\alpha\left(t_{k}\right) \mathbf{l}_{i}\left(t_{k}\right) F\left(t_{k}\right)$, and $\widetilde{Z}_{i}^{\left(q_{l}\right)}\left(u_{l}\right)$ for the term $\alpha\left(u_{l}\right) \mathbf{k}_{i}\left(u_{l}\right) G\left(u_{l}\right)$, where $p_{k}=\rho\left(t_{k}\right)$ and $q_{l}=\rho\left(u_{l}\right)$. Similar notations are used for the terms in $\bar{Y}_{i}^{(p)}, \bar{Z}_{i}^{(p)}, \tilde{y}^{(p)}$, and $\tilde{z}^{(p)}$. Equation (2.8) can be written as

$$
\begin{aligned}
& f\left(y_{0}+\sum_{p=1}^{\infty} \bar{Y}_{i}^{(p)} \frac{h^{p}}{p !}, \sum_{p=1}^{\infty} \widetilde{Z}_{i}^{(p)} \frac{h^{p-1}}{p !}\right)=0 \\
& z_{0}+\sum_{p=1}^{\infty} \bar{Z}_{i}^{(p)} \frac{h^{p}}{p !}=g\left(y_{0}+\sum_{p=1}^{\infty} \bar{Y}_{i}^{(p)} \frac{h^{p}}{p !}\right) .
\end{aligned}
$$

The $n$th derivative of $(2.23)$, evaluated at $h=0$, is

$$
\bar{Z}_{i}^{(n)}=\sum_{\substack{S(u) \in \operatorname{SLDAlT} \\ u=\left[t_{1}, \ldots, t_{k}\right]_{z} \\ \rho(u)=n}} g_{k y}\left(\bar{Y}_{i}^{\left(p_{1}\right)}\left(t_{1}\right), \ldots, \bar{Y}_{i}^{\left(p_{k}\right)}\left(t_{k}\right)\right) .
$$


By use of (2.20), Lemma 2.2, and Definition 2.8 we have

$$
\begin{gathered}
\bar{Z}_{i}^{(n)}=\sum_{\substack{S(u) \in \mathrm{SDA} 1 \mathrm{~T}=\\
u=\left[t_{1}, \ldots, t_{k}\right]_{i} \\
\rho(u)=n}} \beta(S(u)) \alpha\left(t_{1}\right) \cdots \alpha\left(t_{k}\right) \mathbf{v}_{i}\left(t_{1}\right) \cdots \mathbf{v}_{i}\left(t_{k}\right) \\
=\sum_{\substack{S(u) \in \mathrm{SDA} 1 \mathrm{~T} \\
u=\left[t_{1}, \ldots, t_{k}\right]_{z} \\
\rho(u)=n}} \alpha(u) \mathbf{v}_{i}\left(t_{1}\right) \cdots \mathbf{v}_{i}\left(t_{k}\right) G(u) .
\end{gathered}
$$

Comparing this with the expression for $\bar{Z}_{i}^{(n)}$ in (2.20), we obtain

$$
\mathbf{w}_{i}(u)=\mathbf{v}_{i}\left(t_{1}\right) \cdots \mathbf{v}_{i}\left(t_{k}\right), \quad i=1, \ldots, s,
$$

for all $u=\left[t_{1}, \ldots, t_{k}\right]_{z} \in \mathrm{DA}_{1 \mathrm{~T}}$. Multiplying the $(n-1)$ st derivative of $f$ (given by $(2.22)$ ), evaluated at $h=0$, by $\left(-f_{z^{\prime}} g_{y}\right)^{-1}$ gives

$$
\begin{aligned}
& \left.\left(-f_{z^{\prime}} g_{y}\right)^{-1} f^{(n-1)}\right|_{h=0} \\
& =\sum_{\substack{S(t) \in \mathrm{SLDA}_{1 \mathrm{~T}}, t=\left[t_{1}, \ldots, u_{l}\right]_{y} \\
\rho(t)=n}}\left(-f_{z^{\prime}} g_{y}\right)^{-1} f_{k y l z^{\prime}} \\
& \cdot\left(\bar{Y}_{i}^{\left(p_{1}\right)}\left(t_{1}\right), \ldots, \bar{Y}_{i}^{\left(p_{k}\right)}\left(t_{k}\right), \frac{1}{q_{1}} \widetilde{Z}_{i}^{\left(q_{1}\right)}\left(u_{1}\right), \ldots, \frac{1}{q_{l}} \widetilde{Z}_{i}^{\left(q_{l}\right)}\left(u_{l}\right)\right) \\
& +\left(-f_{z^{\prime}} g_{y}\right)^{-1} f_{z^{\prime}} \frac{1}{n} \widetilde{Z}_{i}^{(n)} \\
& =0 \text {. }
\end{aligned}
$$

From (2.10), (2.16), and (2.17) we have

$$
\widetilde{Z}_{i}^{(n)}=\sum_{j=1}^{s} d_{i j} \bar{Z}_{j}^{(n)}=\sum_{j=1}^{s} d_{i j} \sum_{\substack{S(u) \in \mathrm{SLDAIT} \\ u=\left[t_{1}, \ldots, t_{k}\right]_{z} \\ \rho(u)=n}} g_{k y}\left(\bar{Y}_{j}^{\left(p_{1}\right)}\left(t_{1}\right), \ldots, \bar{Y}_{j}^{\left(p_{k}\right)}\left(t_{k}\right)\right) .
$$

Thus, from (2.24),

$$
\begin{aligned}
\left(-f_{z^{\prime}} g_{y}\right)^{-1} f_{z^{\prime}} \widetilde{Z}_{i}^{(n)}= & \sum_{j=1}^{s} d_{i j} \sum_{\substack{S(t) \in{\operatorname{SLDA} 1 T_{y z}}_{t=\left[\left[t_{1}, \ldots, t_{k}\right]_{z}\right]_{y}} \\
\rho(t)=n}}\left(-f_{z^{\prime}} g_{y}\right)^{-1} f_{z^{\prime}} g_{k y}\left(\bar{Y}_{j}^{\left(p_{1}\right)}, \ldots, \bar{Y}_{j}^{\left(p_{k}\right)}\right) \\
& -\sum_{j=1}^{s} d_{i j} \bar{Y}_{j}^{(n)} .
\end{aligned}
$$

Substituting this into (2.26), and using (2.19), (2.20), Lemma 2.2, and Defini- 
tion 2.8, we obtain

$$
\begin{aligned}
\bar{Y}_{i}^{(n)}= & \sum_{\substack{t \in \mathrm{DA}^{\rho} \mathrm{T}_{y} \\
\rho(t)=n}} \mathbf{v}_{i}(t) \alpha(t) F(t) \\
= & n \sum_{j=1}^{s} a_{i j} \sum_{\substack{S(t) \in \mathrm{SDA}^{\prime} \mathrm{S}_{y y} \\
t=\left[t_{1}, \ldots, u_{l}\right]_{y} \\
\rho(t)=n}} \alpha(t) \mathbf{v}_{j}\left(t_{1}\right) \cdots \mathbf{v}_{j}\left(t_{k}\right) \frac{1}{q_{1}} \mathbf{k}_{j}\left(u_{1}\right) \cdots \frac{1}{q_{l}} \mathbf{k}_{j}\left(u_{l}\right) F(t) \\
& +\sum_{\substack{S(t) \in \mathrm{SDA}_{t} \mathrm{~T}_{y=} \\
t=\left[\left[t_{1}, \ldots, t_{k}\right]_{3}\right]_{y} \\
\rho(t)=n}} \alpha(t) \mathbf{v}_{i}\left(t_{1}\right) \cdots \mathbf{v}_{i}\left(t_{k}\right) F(t) .
\end{aligned}
$$

We then have

$$
\begin{array}{r}
\mathbf{v}_{i}(t)=n \sum_{j=1}^{s} a_{i j} \mathbf{v}_{j}\left(t_{1}\right) \cdots \mathbf{v}_{j}\left(t_{k}\right) \frac{1}{\rho\left(u_{1}\right)} \mathbf{k}_{j}\left(u_{1}\right) \cdots \frac{1}{\rho\left(u_{l}\right)} \mathbf{k}_{j}\left(u_{l}\right), \\
i=1, \ldots, s,
\end{array}
$$

if $t=\left[t_{1}, \ldots, t_{k}, u_{1}, \ldots, u_{l}\right]_{y} \in \mathrm{DA}_{1 \mathrm{~T}}, \rho(t)=n$, and

$$
\mathbf{v}_{i}(t)=\mathbf{v}_{i}\left(t_{1}\right) \cdots \mathbf{v}_{i}\left(t_{k}\right), \quad i=1, \ldots, s,
$$

if $t=\left[\left[t_{1}, \ldots, t_{k}\right]_{z}\right]_{y} \in \mathrm{DA} 1 \mathrm{~T}_{y z}$. By using (2.14), (2.15), (2.25), (2.27), and (2.28) we can state the following lemma.

Lemma 2.3. The quantities $Y_{i}, y_{1}$, respectively $Z_{i}$ and $z_{1}, i=1, \ldots, s$, are $\mathrm{DA}_{y}$, respectively $\mathrm{DA}_{z}$, series given by $(2.11)$ and $(2.12)$. The coefficients of these series, and the series of $Y_{i}^{\prime}$ and $Z_{i}^{\prime}$, in (2.13), are given recursively by

$$
\begin{aligned}
& \mathbf{l}_{i}(t)=\rho(t) \mathbf{v}_{i}\left(t_{1}\right) \cdots \mathbf{v}_{i}\left(t_{k}\right) \frac{1}{\rho\left(u_{1}\right)} \mathbf{k}_{i}\left(u_{1}\right), \ldots, \frac{1}{\rho\left(u_{l}\right)} \mathbf{k}_{i}\left(u_{l}\right), \\
& \mathbf{k}_{i}(u)=\sum_{j=1}^{s} d_{i j} \mathbf{v}_{j}\left(t_{1}\right) \cdots \mathbf{v}_{j}\left(t_{k}\right)
\end{aligned}
$$

for $t=\left[t_{1}, \ldots, t_{k}, u_{1}, \ldots, u_{l}\right]_{y} \in \mathrm{DA} 1 \mathrm{~T}_{y}$, and $u=\left[t_{1}, \ldots, t_{k}\right]_{z} \in \mathrm{DA} 1 \mathrm{~T}_{z}$, and by

$$
\mathbf{v}_{i}(t)=\sum_{j=1}^{s} a_{i j} \mathbf{l}_{j}(t), \quad \mathbf{w}_{i}(u)=\sum_{j=1}^{s} a_{i j} \mathbf{k}_{j}(u)
$$

and

$$
\mathbf{y}_{1}(t)=\sum_{i=1}^{s} b_{i} \mathbf{l}_{i}(t), \quad \mathbf{z}_{1}(u)=\sum_{i=1}^{s} b_{i} \mathbf{k}_{i}(u),
$$

where $\mathbf{l}_{i}(\varnothing)=0, \mathbf{k}_{i}(\varnothing)=0$, and

$$
\mathbf{l}_{i}\left(\tau_{y}\right)=1, \quad \mathbf{k}_{i}\left(\tau_{z}\right)=1 .
$$


Note that the coefficients $\mathbf{l}_{i}(t)$ and $\mathbf{k}_{i}(u)$ can be written as

$$
\begin{aligned}
\mathbf{l}_{i}(t)=\rho(t) \frac{1}{\rho\left(u_{1}\right)} \cdots \frac{1}{\rho\left(u_{l}\right)} \sum_{n_{1}, \ldots, n_{k}=1}^{s} a_{i n_{1}} \cdots a_{i n_{k}} \\
\cdot \mathbf{l}_{n_{1}}\left(t_{1}\right) \cdots \mathbf{l}_{n_{k}}\left(t_{k}\right) \mathbf{k}_{i}\left(u_{1}\right) \cdots \mathbf{k}_{i}\left(u_{l}\right)
\end{aligned}
$$

for $t=\left[t_{1}, \ldots, t_{k}, u_{1}, \ldots, u_{l}\right]_{y} \in \mathrm{DA} 1 \mathrm{~T}_{y}$, and

$$
\mathbf{k}_{i}(u)=\sum_{j=1}^{s} d_{i j} \sum_{n_{1}, \ldots, n_{k}=1}^{s} a_{j n_{1}} \cdots a_{j n_{k}} \mathbf{l}_{n_{1}}\left(t_{1}\right) \cdots \mathbf{l}_{n_{k}}\left(t_{k}\right)
$$

for $u=\left[t_{1}, \ldots, t_{k}\right]_{z}$. Equation (2.32) can be proved by inserting (2.16) into (2.8) and using the Taylor expansions of $f$ and $g$. We can then express (2.8) as power series in $h$. The first terms in these expressions will give us $f\left(y, g_{y} \tilde{Y}_{i}^{\prime}\right)=$ 0 and $\tilde{Z}_{i}^{\prime}=g_{y} \tilde{Y}_{i}^{\prime}$, so that

$$
\tilde{Y}_{i}^{\prime}=y^{\prime} \text { and } \quad \tilde{Z}_{i}^{\prime}=z^{\prime}
$$

Comparing this with (2.19), we have that

$$
\tilde{Y}_{i}^{\prime}=\mathbf{l}_{i}\left(\tau_{y}\right) F\left(\tau_{y}\right)=y^{\prime} \Rightarrow \mathbf{l}_{i}\left(\tau_{y}\right)=1 .
$$

Therefore, $\mathbf{k}_{i}\left(\tau_{z}\right)$ is given by (2.29) and (2.30).

We observe that $\mathbf{y}_{1}(t)$ and $z_{1}(t)$ can be written as $\gamma(t) \Phi(t)$, where $\gamma(t)$ is a rational number and $\Phi(t)$ is some combination of the method coefficients. The number $\gamma(t)$ is given by the definition below, and $\Phi(t)$ can be read directly from the tree, by the following procedure. Let $t \in \mathrm{DA} 1 \mathrm{~T}$. To the root of the tree, attach the label $i$ if the root is light and $j$ if the root is heavy. To the other vertices, attach other labels, say $k, l, m, \ldots$. For each arc write down the factor $a_{v w}$ if the succeeding vertex (labelled $w$ ) is light, $v, w$ being the labels at the end of the arc. Similarly, write down the factor $d_{v w}$ if the succeeding vertex is a heavy vertex. Insert a further factor $b_{i}$ if $t \in \mathrm{DA}_{1 \mathrm{~T}}$, and $b_{i} d_{i j}$ if $t \in \mathrm{DA}_{z}$, and sum over each index $i, j, \ldots, k$ in the range from 1 to $s$. The sum is $\Phi(t)$ and is called the elementary weight for the tree $t$. To each tree we also associate the following rational number:

Definition 2.10. Let $\gamma(t):$ DA1T $\rightarrow \mathbf{Q}$, where $\mathbf{Q}$ is the set of rational numbers, be defined recursively by

$$
\begin{gathered}
\gamma\left(\tau_{y}\right)=1, \quad \gamma\left(\tau_{z}\right)=1, \\
\gamma(t)=\rho(t) \frac{1}{\rho\left(u_{1}\right)} \cdots \frac{1}{\rho\left(u_{l}\right)} \cdot \gamma\left(t_{1}\right) \cdots \gamma\left(t_{k}\right) \gamma\left(u_{1}\right) \cdots \gamma\left(u_{l}\right)
\end{gathered}
$$

for $t=\left[t_{1}, \ldots, t_{k}, u_{1}, \ldots, u_{l}\right]_{y} \in \mathrm{DA} 1 \mathrm{~T}_{y}$, and

$$
\gamma(u)=\gamma\left(t_{1}\right) \cdots \gamma\left(t_{k}\right)
$$

for $u=\left[t_{1}, \ldots, t_{k}\right]_{z} \in \mathrm{DAlT}_{z}$. 
Example 2.4. Let $t \in \mathrm{DA}_{1} \mathrm{~T}_{y}$ and $u \in \mathrm{DA} 1 \mathrm{~T}_{z}$. Their elementary weights and rational numbers are given by

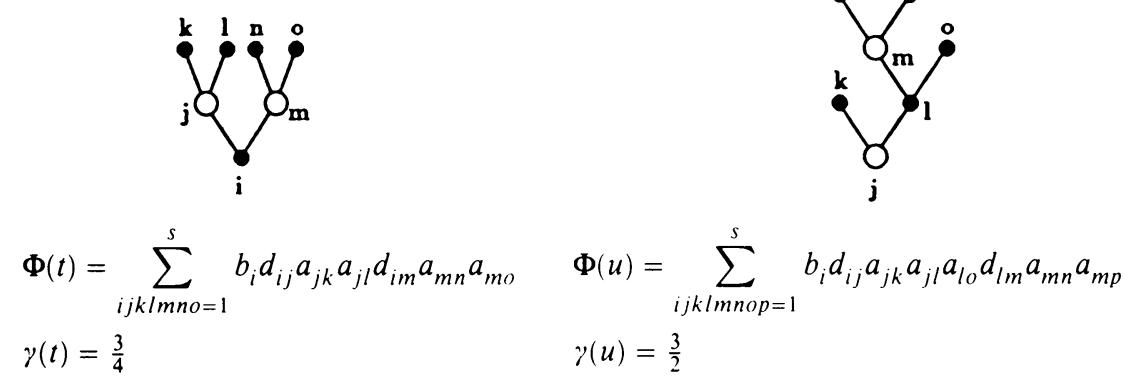

The order conditions for Runge-Kutta methods applied to DAE problems are given by the following theorem.

Theorem 2.2. If the method (1.2), (1.3) is applied to the problem (2.1), then the order of the local truncation error is $p+1$ if and only if

$$
\Phi(t)=\frac{1}{\gamma(t)}
$$

for all $t \in \mathrm{DA} 1 \mathrm{~T}$ with $\rho(t) \leq p$.

Proof. The Taylor expansion of the exact solution of (2.1) can be written as the DAl series

$$
y\left(x_{0}+h\right)=\mathrm{DA}_{y}\left(\mathbf{p}_{y}, y_{0}, z_{0}\right), \quad z\left(x_{0}+h\right)=\mathrm{DA}_{z}\left(\mathbf{p}_{z}, y_{0}, z_{0}\right)
$$

with $\mathbf{p}_{y}, \mathbf{p}_{z}=1$ for all $t \in \mathrm{DA} 1 \mathrm{~T}$. By comparing (2.36) term by term with the DAl series (2.12) for the numerical solution, and by using (2.33), (2.34), and Lemma 2.3, we have proved Theorem 2.2 for the model equation (2.1). Theorem 1.1 shows that this result is also valid for the general index-1 problem.

2.3. Simplification of Theorem 2.2. Let $t \in \mathrm{DA} 1 \mathrm{~T}_{y}$; then $t$ can be associated with a simplified tree $\bar{t}$ as follows: If a heavy vertex has no ramifications, and is followed by a light vertex, then the tree can be simplified by removing these two vertices. Similarly, if a light vertex (except the root) with no ramifications is followed by a heavy vertex, the tree can be simplified by removing these two vertices. The simplified tree $\bar{t}$ corresponding to $t$ is the tree which is simplified as much as possible.

Example 2.5. The figure shows a tree $t$ and its corresponding simplified tree $\bar{t}$.

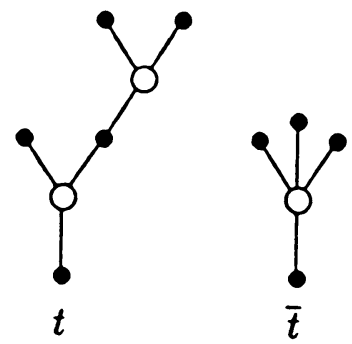


The set of simplified trees $\bar{t}$ is defined recursively by

Definition 2.11. The sets $\overline{\mathrm{DA}_{1} \mathrm{~T}_{y}} \subset \mathrm{DA} \mathrm{T}_{y}$ and $\overline{\mathrm{DA}_{\mathrm{T}}} \subset \mathrm{DA} 1 \mathrm{~T} z$ of simplified trees $\bar{t}$ are defined recursively as follows:

1. $\tau_{y} \in \overline{\mathrm{DA}^{2} \mathrm{~T}_{y y}}$.

2. (a) If $\bar{t}_{1}, \ldots, \bar{t}_{k} \in \overline{\mathrm{DAIT}_{y y}}$ and $k>1$, then $\left[\bar{t}_{1}, \ldots, \bar{t}_{k}\right]_{z} \in \overline{\mathrm{DA} \mathrm{TT}_{z}}$.

(b) If $\bar{t}_{1}, \ldots, \bar{t}_{k} \in \overline{\mathrm{DA}_{\mathrm{DT}} y}, \bar{u}_{1}, \ldots, \bar{u}_{l} \in \overline{\mathrm{DA}_{1 \mathrm{~T}}}, k>0$ or $k=0$,

$l>1$, then $\left[\bar{t}_{1}, \ldots, \bar{t}_{k}, \bar{u}_{1}, \ldots, \bar{u}_{l}\right]_{y} \in \overline{\mathrm{DA} 1 \mathrm{~T}}_{y y}$.

(c) If $\bar{u} \in \overline{\mathrm{DA}_{1 \mathrm{~T}}}$, then $[\bar{u}]_{y} \in \overline{\mathrm{DA}_{1 \mathrm{~T}}}$.

3. $\overline{\mathrm{DA}_{1} \mathrm{~T}_{y}}=\overline{\mathrm{DA}_{1 \mathrm{~T}}} \cup \overline{\mathrm{DA}_{y y} \mathrm{~T}_{y z}}$.

Theorem 2.3. To each tree $t \in \mathrm{DA} 1 \mathrm{~T}$ there is a corresponding simplified tree $\bar{t} \in \overline{\mathrm{DA}^{2} \mathrm{~T}_{y}}$ such that $\Phi(t)=\Phi(\bar{t})$ and $\gamma(t)=\gamma(\bar{t})$.

Before we prove this theorem, we will give an example.

Example 2.6. Consider the tree $t$ and the corresponding simplified tree $\bar{t}$ given by Example 2.5. Their elementary weights are given by

$$
\Phi(t)=\sum_{i, j, k, l=1}^{s} b_{i} d_{i j} c_{j} a_{j k} d_{k l} c_{l}^{2}, \quad \Phi(\bar{t})=\sum_{i, j=1}^{s} b_{i} d_{i j} c_{j}^{3} .
$$

By using the fact that

$$
\sum_{k=1}^{s} a_{j k} d_{k l}=\delta_{j l}= \begin{cases}1 & \text { if } j=l \\ 0 & \text { otherwise }\end{cases}
$$

we have that $\Phi(t)=\Phi(\bar{t})$. In addition, we have

$$
\gamma(t)=3 \cdot\left(\frac{1}{3} \cdot\left(1 \cdot 2 \cdot\left(\frac{1}{2} \cdot(1 \cdot 1)\right)\right)\right)=1, \quad \gamma(\bar{t})=3 \cdot\left(\frac{1}{3} \cdot(1 \cdot 1 \cdot 1)\right)=1,
$$

in agreement with the statement of the theorem.

Proof of Theorem 2.3. Let

$$
t=\left[\bar{t}_{1}, \ldots, \bar{t}_{k}, t_{p}, \bar{u}_{1}, \ldots, \bar{u}_{l}, u_{q}\right]_{y} \in \mathrm{DA}_{1} \mathrm{~T}_{y},
$$

where

$$
\begin{aligned}
& t_{p}=\left[\bar{u}_{p}\right]_{y}=\left[\left[\bar{t}_{p, 1}, \ldots, \bar{t}_{p, k_{p}}\right]_{z}\right]_{y} \in \overline{\mathrm{DA} 1 \mathrm{~T}_{y z}}, \\
& u_{q}=\left[\bar{t}_{q}\right]_{z}=\left[\left[\bar{t}_{q, 1}, \ldots, \bar{t}_{q, k_{q}}, \bar{u}_{q, 1}, \ldots, \bar{u}_{q, l_{q}}\right]_{y}\right]_{z} \in \mathrm{DA} 1 \mathrm{~T} z y \text {, } \\
& \bar{t}_{1}, \ldots, \bar{t}_{k}, \bar{t}_{q}, \bar{t}_{p, 1}, \ldots, \bar{t}_{p, k_{p}}, \bar{t}_{q, 1}, \ldots, \bar{t}_{q, k_{q}} \in \overline{\mathrm{DA} \mathrm{DT}_{y y}}, \\
& \bar{u}_{1}, \ldots, \bar{u}_{l}, \bar{u}_{p}, \bar{u}_{q, 1}, \ldots, \bar{u}_{q, l_{q}} \in \overline{\mathrm{DA} \mathrm{DT}_{z}} \text {. }
\end{aligned}
$$

From (2.33), (2.34), and Definition 2.7 we have

$$
\begin{aligned}
\mathbf{l}_{j}\left(t_{p}\right) & =\rho\left(t_{p}\right) \frac{1}{\rho\left(\bar{u}_{p}\right)} \mathbf{k}_{j}\left(\bar{u}_{p}\right) \\
& =\sum_{m=1}^{s} d_{j m} \sum_{N_{1}, \ldots, N_{k_{p}}=1}^{s} a_{m N_{1}} \cdots a_{m N_{k_{p}}} \mathbf{l}_{N_{1}}\left(t_{p, 1}\right) \cdots \mathbf{l}_{N_{k_{p}}}\left(t_{p, k_{p}}\right),
\end{aligned}
$$




$$
\begin{aligned}
\mathbf{k}_{i}\left(u_{q}\right)= & \sum_{j=1}^{s} d_{i j} \sum_{m=1}^{s} a_{j m} \mathbf{l}_{m}\left(\bar{t}_{q}\right)=\mathbf{l}_{i}\left(\bar{t}_{q}\right) \\
= & \rho\left(\bar{t}_{q}\right) \frac{1}{\rho\left(\bar{u}_{q, 1}\right) \cdots \rho\left(\bar{u}_{q, l_{q}}\right)} \\
& \cdot \sum_{M_{1}, \ldots, M_{k_{q}}=1}^{s} a_{i M_{1}} \cdots a_{i M_{k_{q}}} \mathbf{l}_{M_{1}}\left(\bar{t}_{q, 1}\right) \cdots \mathbf{k}_{i}\left(\bar{u}_{q, l_{q}}\right) .
\end{aligned}
$$

By using the fact that $\rho\left(u_{q}\right)=\rho\left(\bar{t}_{q}\right)$ we have

$$
\mathbf{l}_{i}(t)=\Gamma(t) \sum_{\substack{n_{1}, \ldots, n_{k}=1 \\ N_{1}, \ldots, N_{k_{p}}=1 \\ M_{1}, \ldots, M_{k_{q}}=1}}^{s} a_{i n_{1}} \cdots a_{i n_{k}} a_{i N_{1}} \cdots a_{i N_{k_{p}}} a_{i M_{1}} \cdots a_{i M_{k_{q}}} \mathscr{L}(t) \mathscr{K}(t),
$$

where

$$
\begin{gathered}
\Gamma(t)=\rho(t) \frac{1}{\rho\left(\bar{u}_{1}\right)} \cdots \frac{1}{\rho\left(\bar{u}_{l}\right)} \frac{1}{\rho\left(\bar{u}_{q, 1}\right)} \cdots \frac{1}{\rho\left(\bar{u}_{q, l_{q}}\right)}, \\
\mathscr{L}(t)=\mathbf{l}_{n_{1}}\left(\bar{t}_{1}\right) \cdots \mathbf{l}_{n_{k}}\left(\bar{t}_{k}\right) \mathbf{l}_{N_{1}}\left(\bar{t}_{p, 1}\right) \cdots \mathbf{l}_{N_{k_{p}}}\left(\bar{t}_{p, k_{p}}\right) \mathbf{l}_{M_{1}}\left(\bar{t}_{q, 1}\right) \cdots \mathbf{l}_{M_{k_{q}}}\left(\bar{t}_{q, k_{q}}\right),
\end{gathered}
$$

and

$$
\mathscr{K}(t)=\mathbf{k}_{i}\left(\bar{u}_{1}\right) \cdots \mathbf{k}_{i}\left(\bar{u}_{l}\right) \mathbf{k}_{i}\left(\bar{u}_{q, 1}\right) \cdots \mathbf{k}_{i}\left(\bar{u}_{q, l_{q}}\right) .
$$

Therefore,

$$
\mathbf{l}_{i}(t)=\mathbf{l}_{i}(\bar{t})
$$

where

$$
\begin{aligned}
\bar{t}=\left[\bar{t}_{1}, \ldots, \bar{t}_{k}, \bar{t}_{p, 1}, \ldots, \bar{t}_{p, k_{p}}, \bar{t}_{q, 1}, \ldots, \bar{t}_{q, k_{q}},\right. \\
\left.\bar{u}_{1}, \ldots, \bar{u}_{l}, \bar{u}_{q, 1}, \ldots, \bar{u}_{q, l_{q}}\right]_{y} \in \overline{\mathrm{DA} 1 \mathrm{~T}}_{y y} .
\end{aligned}
$$

It is obvious that this result is valid even if the tree $t$ consists of more (or less) than one subtree $t \in \overline{\mathrm{DA}}_{y z}$ and more (or less) than one $u \in \mathrm{DA} \mathrm{DT}_{z y}$. Similarly, if $u=\left[\bar{t}_{1}, \ldots, \bar{t}_{k}, t_{p}\right]_{z}$, where $t_{p}=\left[\bar{u}_{p}\right]_{y}=\left[\left[\bar{t}_{p, 1}, \ldots, \bar{t}_{p, k_{p}}\right]_{z}\right]_{y} \in$ $\overline{\mathrm{DA} 1 \mathrm{~T}}_{y z}$ and $\bar{t}_{1}, \ldots, \bar{t}_{k}, \bar{t}_{p, 1}, \ldots, \bar{t}_{p, k_{p}} \in \overline{\mathrm{DA} 1 \mathrm{~T}}_{y y}$, then

$$
\mathbf{k}_{i}(u)=\mathbf{k}_{i}(\bar{u})
$$

where

$$
\bar{u}=\left[\bar{t}_{1}, \ldots, \bar{t}_{l}, \bar{t}_{p, 1}, \ldots, \bar{t}_{p, l_{p}}\right]_{z} \in \overline{\mathrm{DA}_{1 \mathrm{~T}}} \text {. }
$$

If $t=[u]_{y} \in \mathrm{DAlT}_{y z}$, then $\bar{t}=[\bar{u}]_{y z}$. By repeated use of (2.38) and (2.41) we can show that for all $t \in \mathrm{DA}_{1} \mathrm{~T}_{y}$ and for all $u \in \mathrm{DA}_{1 \mathrm{~T}}$ there exist corresponding simplified trees $\bar{t} \in \overline{\mathrm{DA} 1 \mathrm{~T}}_{y}$ and $\bar{u} \in \overline{\mathrm{DA} 1 \mathrm{~T}}_{z}$ such that $\mathbf{l}_{i}(t)=\mathbf{l}_{i}(\bar{t})$ and $\mathbf{k}_{i}(u)=\mathbf{k}_{i}(\bar{u})$. From (2.37) we have

$$
\mathbf{l}_{i}(\bar{t})=\mathbf{k}_{i}(\bar{u})=\mathbf{k}_{i}(u) \quad \text { if } t=[u]_{y} \in \mathrm{DA} 1 \mathrm{~T}_{y z} .
$$


Inserting this into (2.31), we get $\mathbf{y}_{1}(t)=\mathbf{y}_{1}(\bar{t})$ and $\mathbf{z}_{1}(u)=\mathbf{y}_{1}(\bar{t})$, where $\bar{t}=$ $[\bar{u}]_{y} \in \overline{\mathrm{DA} 1 \mathrm{~T}}_{y z}$. Theorem 2.3 is proved.

In Figure 2 we give the order condition related to the trees up to order 4.

$\rho(t) \quad t \quad \Phi(t)=\frac{1}{\gamma(t)}$

1

$\sum_{i} b_{i}=1$

2

$\sum_{i} b_{i} c_{i}=\frac{1}{2}$

2

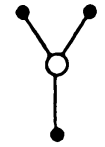

$\sum_{i j} b_{i} d_{i j} c_{j}^{2}=1$

3

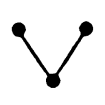

$\sum_{i} b_{i} c_{i}^{2}=\frac{1}{3}$

3

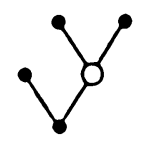

$\sum_{i j} b_{i} c_{i} d_{i j} c_{j}^{2}=\frac{2}{3}$

3

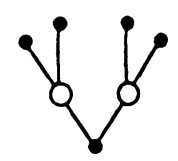

$\sum_{i j k} b_{i} d_{i j} c_{j}^{2} d_{i k} c_{k}^{2}=\frac{4}{3}$

3

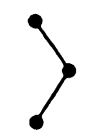

$\sum_{i j} b_{i} a_{i j} c_{j}=\frac{1}{6}$

3

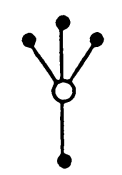

$\sum_{i j} b_{i} d_{i j} c_{j}^{3}=1$

3

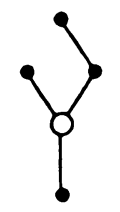

$\sum_{i j k} b_{i} d_{i j} c_{j} a_{j k} c_{k}=\frac{1}{2}$

4

$\sum_{i} b_{i} c_{i}^{3}=\frac{1}{4}$ 


$$
\rho(t) \quad t \quad \Phi(t)=\frac{1}{\gamma(t)}
$$

4

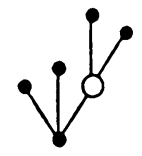

$\sum_{i j} b_{i} c_{i}^{2} d_{i j} c_{j}^{2}=\frac{1}{2}$

4

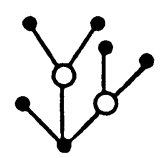

$\sum_{i j k} b_{i} c_{i} d_{i j} c_{j}^{2} d_{i k} c_{k}^{2}=1$

4

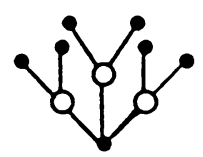

$\sum_{i j k l} b_{i} d_{i j} c_{j}^{2} d_{i k} c_{k}^{2} d_{i l} c_{l}^{2}=2$

4

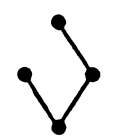

$\sum_{i j} b_{i} c_{i} a_{i j} c_{j}=\frac{1}{8}$

4

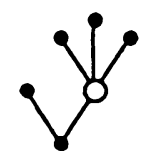

$\sum_{i j} b_{i} c_{i} d_{i j} c_{. j}^{3}=\frac{3}{4}$

4

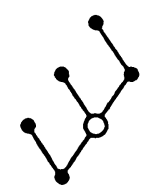

$\sum_{i j k} b_{i} c_{i} d_{i j} c_{j} a_{j k} c_{k}=\frac{3}{8}$

4

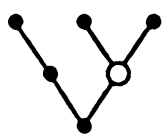

$\sum_{i j k} b_{i} a_{i j} c_{j} d_{i k} c_{k}^{2}=\frac{1}{4}$

4

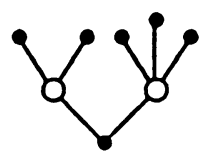

$\sum_{i j k} b_{i} d_{i j} c_{j}^{2} d_{i k} c_{k}^{3}=\frac{3}{2}$

4

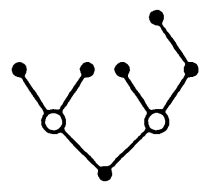

$\sum_{i j k l} b_{i} d_{i j} c_{j}^{2} d_{i k} c_{k} a_{k l} c_{l}=\frac{3}{4}$ 


$\rho(t) \quad t \quad \Phi(t)=\frac{1}{\gamma(t)}$

4

$\sum_{i j} b_{i} a_{i j} c_{j}^{2}=\frac{1}{12}$

4

$\sum_{i j k} b_{i} a_{i j} c_{j} d_{j k} c_{k}^{2}=\frac{1}{6}$

4

$\sum_{i j k l} b_{i} a_{i j} d_{j k} c_{k}^{2} d_{j l} c_{l}^{2}=\frac{1}{3}$

4

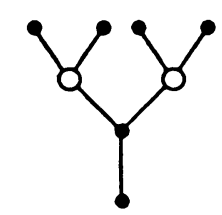

$\sum_{i j k} b_{i} a_{i j} a_{j k} c_{k}=\frac{1}{24}$

4

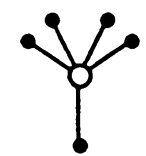

$\sum_{i j} b_{i} d_{i j} c_{j}^{4}=1$

4

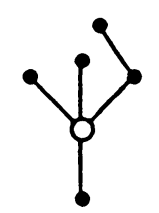

$\sum_{i j k} b_{i} d_{i j} c_{j}^{2} a_{j k} c_{k}=\frac{1}{2}$

4

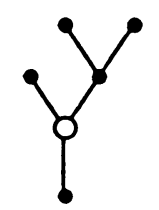

$$
\sum_{i j k} b_{i} d_{i j} c_{j} a_{j k} c_{k}^{2}=\frac{1}{3}
$$

4

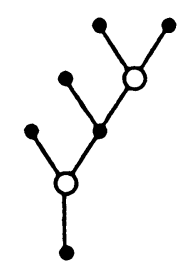

$\sum_{i j k l} b_{i} d_{i j} c_{j} a_{j k} c_{k} d_{k l} c_{l}^{2}=\frac{2}{3}$ 


$\rho(t) \quad t \quad \Phi(t)=\frac{1}{\gamma(t)}$

4

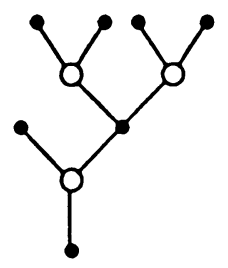

$$
\sum_{i j k l m} b_{i} d_{i j} c_{j} a_{j k} d_{k l} c_{l}^{2} d_{k m} c_{m}^{2}=\frac{4}{3}
$$

4

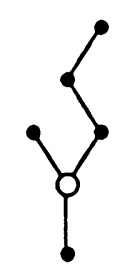

$$
\sum_{i j k l} b_{i} d_{i j} c_{j} a_{j k} a_{k l} c_{l}=\frac{1}{6}
$$

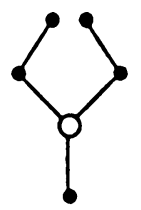

$$
\sum_{i j k l} b_{i} d_{i j} a_{j k} c_{k} a_{k l} c_{l}=\frac{1}{4}
$$

Figure 2. Order conditions related to trees.

\section{CONVERgence Results}

This section deals with the convergence of a Runge-Kutta method applied to the differential-algebraic equation (1.6). The system is assumed to be a uniform index-1 problem. We first give some preliminary results about the existence of a solution of the Runge-Kutta equations, and the influence of perturbations to the solution of these equations. The convergence results are given in $\S 3.2$.

3.1. Preliminary results. The results of the next two theorems are essentially the same as those given by Hairer et al. [7] for the index-1 component of a Hessenberg form DAE of size 2.

3.1.1. The existence of a Runge-Kutta solution.

Theorem 3.1. Let $(\nu, \zeta)$ satisfy

$$
f(\nu, \zeta)=\mathscr{O}(h), \quad g(\nu)=\mathscr{O}\left(h^{2}\right) .
$$

Let the coefficients of the method satisfy

$$
f\left(\tilde{\nu}_{i}, \zeta\right)=\mathscr{O}(h), \quad g\left(\tilde{\nu}_{i}\right)=\mathscr{O}\left(h^{2}\right), \quad i=1, \ldots, s,
$$


where $\tilde{\nu}_{i}=\nu+c_{i} h \zeta$ and $c_{i}=\sum_{j=1}^{s} a_{i j}$. Suppose that

$$
\left\|\left[\begin{array}{l}
f_{v^{\prime}} \\
g_{v}
\end{array}\right]^{-1}\right\| \leq M_{1} \text { and }\left\|f_{v}\right\| \leq M_{2}
$$

in an $h$-independent neighborhood of $(\nu, \zeta)$. If the coefficient matrix $\mathscr{A}$ of the method is invertible, then there exists a solution of (1.2), (1.4) which satisfies

$$
V_{i}^{\prime}=\zeta+\mathscr{O}(h), \quad V_{i}=\nu+\mathscr{O}(h) .
$$

Proof. Consider the homotopy

$$
\begin{aligned}
& f\left(\nu+h \sum_{j=1}^{s} a_{i j} V_{j}^{\prime}, V_{i}^{\prime}\right)=(1-\tau) f\left(\tilde{\nu}_{i}, \zeta\right), \\
& g\left(\nu+h \sum_{j=1}^{s} a_{i j} V_{j}^{\prime}\right)=(1-\tau) g\left(\tilde{\nu}_{i}\right), \\
& V_{i}=\nu+h \sum_{j=1}^{s} a_{i j} V_{j}^{\prime} .
\end{aligned}
$$

For $\tau=0$, this system has the solution $V_{i}^{\prime}=\zeta, V_{i}=\tilde{\nu}_{i}$. For $\tau=1$ it is equivalent to (1.2), (1.4). We consider $V_{i}^{\prime}$ as functions of $\tau$, and differentiate (3.3) with respect to this parameter:

$$
\begin{aligned}
& f_{v^{\prime}}\left(V_{i}, V_{i}^{\prime}\right) \dot{V}_{i}^{\prime}+f_{v}\left(V_{i}, V_{i}^{\prime}\right) h \sum_{j=1}^{s} a_{i j} \dot{V}_{j}^{\prime}=-f\left(\tilde{\nu}_{i}, \zeta\right), \\
& g_{v}\left(V_{i}\right) h \sum_{j=1}^{s} a_{i j} \dot{V}_{j}^{\prime}=-g\left(\tilde{\nu}_{i}\right),
\end{aligned}
$$

Dividing the second equation by $h$, the system can be written in matrix form as follows:

$$
\left[\begin{array}{c}
\left\{f_{v^{\prime}}\right\}+h\left\{f_{v}\right\}\left(\mathscr{A} \otimes I_{m}\right) \\
\left\{g_{v}\right\}\left(\mathscr{A} \otimes I_{m}\right)
\end{array}\right] \dot{V}^{\prime}=\left[\begin{array}{c}
-\tilde{f}(\nu, \zeta) \\
-\frac{1}{h} \tilde{g}(\nu)
\end{array}\right] .
$$

Here, $\left\{f_{v^{\prime}}\right\},\left\{f_{v}\right\}$, and $\left\{g_{v}\right\}$ are block-diagonal matrices:

$$
\left\{f_{v^{\prime}}\right\}=\operatorname{blockdiag}\left[f_{v^{\prime}}\left(V_{1}, V_{1}^{\prime}\right), \ldots, f_{v^{\prime}}\left(V_{s}, V_{s}^{\prime}\right)\right],
$$

etc. Furthermore, $\dot{V}^{\prime}=\left[\dot{V}_{1}^{T}, \ldots, \dot{V}_{s}^{T}\right]^{T}, I_{m}$ is the $m \times m$ identity matrix, and $\tilde{f}(\nu, \zeta)$ and $\tilde{g}(\nu, \zeta)$ are

$$
\left[f^{T}\left(\tilde{\nu}_{1}, \zeta\right), \ldots, f^{T}\left(\tilde{\nu}_{s}, \zeta\right)\right]^{T} \text { and }\left[g^{T}\left(\tilde{\nu}_{1}\right), \ldots, g^{T}\left(\tilde{\nu}_{s}\right)\right]^{T},
$$

resp. We have $g_{v}\left(V_{i}\right) a_{i j}=a_{i j} g_{v}\left(V_{j}\right)+\mathscr{O}(d)$, provided that $\left\|V_{i}-V_{j}\right\| \leq d, d$ independent of $h$. Then the coefficient matrix of (3.4) can be written as

$$
\left[\begin{array}{c}
\left\{f_{v^{\prime}}\right\}+\mathscr{O}(h) \\
\left(\mathscr{A} \otimes I_{m}\right)\left\{g_{v}\right\}+\mathscr{O}(d)
\end{array}\right]
$$


and (3.4) becomes

$$
\left[\begin{array}{l}
\left\{f_{v^{\prime}}\right\}+\mathscr{O}(h) \\
\left\{g_{v}\right\}+\mathscr{O}(d)
\end{array}\right] \dot{V}^{\prime}=\left[\begin{array}{c}
-\tilde{f}(\nu, \zeta) \\
-\frac{1}{h}\left(\mathscr{A} \otimes I_{m}\right)^{-1} \tilde{g}(\nu)
\end{array}\right] .
$$

The matrix here has a bounded inverse, provided that $d$ and $h$ are sufficiently small. Using the assumption (3.1), we have $\dot{V}^{\prime}=\mathscr{O}(h)$ and

$$
V_{i}^{\prime}=\zeta+\int_{0}^{\tau} \dot{V}_{i}^{\prime}(t) d t=\zeta+\mathscr{O}(h)
$$

This shows that all $V_{i}^{\prime}(\tau)$ remain in a small, $h$-independent neighborhood of $\zeta$ for all $|\tau| \leq 1$ and for sufficiently small $h$. Hence, the differential equation (3.4) with initial values $V^{\prime}(0)=\varepsilon_{s} \otimes \zeta$ possesses a solution at least for $0 \leq \tau \leq 1$. This proves the existence of a solution $V_{i}^{\prime}$ of (1.2) and also the first estimate of (3.2). The estimate for $V_{i}$ follows directly from (1.4).

\subsubsection{The influence of perturbations.}

Theorem 3.2. Let $V_{i}^{\prime}, V_{i}$ be given by (1.2), (1.4) and consider perturbed values $\widehat{V}_{i}^{\prime}, \widehat{V}_{i}$ satisfying

$$
\begin{aligned}
& f\left(\widehat{V}_{i}, \widehat{V}_{i}^{\prime}\right)+\delta_{i}=0, \\
& g\left(\widehat{V}_{i}\right)+\theta_{i}=0, \\
& \widehat{V}_{i}=\hat{\nu}+h \sum_{j=1}^{s} a_{i j} \widehat{V}_{j}^{\prime},
\end{aligned}
$$

In addition to the hypotheses of Theorem 3.1, assume that

$$
\hat{\nu}-\nu=\mathscr{O}\left(h^{2}\right), \quad \delta_{i}=\mathscr{O}(h), \quad \theta_{i}=\mathscr{O}\left(h^{2}\right) .
$$

Then, for $h \leq h_{0}$, we have the estimate

$$
\begin{aligned}
\left\|\widehat{V}_{i}^{\prime}-V_{i}^{\prime}\right\|_{\infty} \leq C\left(\frac{1}{h}\left\|g_{v}(\nu) \cdot(\hat{\nu}-\nu)\right\|_{\infty}\right. & +\left\|f_{v}(\nu, \zeta) \cdot(\hat{\nu}-\nu)\right\|_{\infty} \\
& \left.+\|\hat{\nu}-\nu\|_{\infty}+\|\delta\|_{\infty}+\frac{1}{h}\|\theta\|_{\infty}\right),
\end{aligned}
$$

where $\delta=\left[\delta_{1}^{T}, \ldots, \delta_{s}^{T}\right]^{T}$ and $\theta=\left[\theta_{1}^{T}, \ldots, \theta_{s}^{T}\right]^{T}$.

Proof. Consider the homotopy

$$
\begin{aligned}
& f\left(V_{i}, V_{i}^{\prime}\right)+(1-\tau) \delta_{i}=0, \\
& g\left(V_{i}\right)+(1-\tau) \theta_{i}=0, \\
& V_{i}=\nu+h \sum_{j=1}^{s} a_{i j} V_{j}^{\prime}+(1-\tau)(\hat{\nu}-\nu),
\end{aligned} \quad i=1, \ldots, s .
$$

For $\tau=1$, this system is equivalent to (1.2), (1.4); for $\tau=0$, it is equivalent to the perturbed system (3.7). As before, $V_{i}$ and $V_{i}^{\prime}$ are considered as functions 
of $\tau$, and (3.10) is differentiated with respect to this parameter. We then obtain

$$
\begin{aligned}
& f_{v^{\prime}}\left(V_{i}, V_{i}^{\prime}\right) \dot{V}_{i}^{\prime}+f_{v}\left(V_{i}, V_{i}^{\prime}\right) \dot{V}_{i}-\delta_{i}=0, \\
& g_{v}\left(V_{i}\right) \dot{V}_{i}-\theta_{i}=0, \\
& \dot{V}_{i}=h \sum_{j=1}^{s} a_{i j} \dot{V}_{j}^{\prime}-(\hat{\nu}-\nu),
\end{aligned} \quad i=1, \ldots, s .
$$

Inserting the last equation into the other two and using as before

$$
g_{v}\left(V_{i}\right) a_{i j}=a_{i j} g_{v}\left(V_{j}\right)+\mathscr{O}(d) \text {, }
$$

we have

$$
\left[\begin{array}{l}
\left\{f_{v^{\prime}}\right\}+\mathscr{O}(h) \\
\left\{g_{v}\right\}+\mathscr{O}(d)
\end{array}\right] \dot{V}^{\prime}=\left[\begin{array}{c}
\left\{f_{v}\right\}\left(\varepsilon_{s} \otimes(\hat{\nu}-\nu)\right)+\delta \\
\frac{1}{h}\left(\mathscr{A} \otimes I_{m}\right)^{-1}\left(\left\{g_{v}\right\}\left(\varepsilon_{s} \otimes(\hat{\nu}-\nu)\right)+\theta\right)
\end{array}\right] .
$$

As in the proof of Theorem 3.1, the matrix is nonsingular for $h$ and $d$ sufficiently small, and we have

$$
\begin{aligned}
\left\|\dot{V}^{\prime}\right\|_{\infty} \leq & C_{1}\left(\left\|f_{v}(\hat{\nu}-\nu)\right\|_{\infty}+\|\delta\|_{\infty}+h\|(\hat{\nu}-\nu)\|_{\infty}\right) \\
& +\frac{C_{2}}{h}\left(\left\|g_{v}(\hat{\nu}-\nu)\right\|_{\infty}+\|\theta\|_{\infty}+h\|(\hat{\nu}-\nu)\|_{\infty}\right) .
\end{aligned}
$$

The term $h\|(\hat{\nu}-\nu)\|_{\infty}$ comes from the $\mathscr{O}(h)$ term in the matrix of (3.11). Now, by (3.6), the assertion of the theorem is proved.

For the special case where $\delta=\theta=0$ and $\hat{\nu}-\nu=\mathscr{O}(h)$ we have

$$
\left\|\hat{V}_{i}^{\prime}-V_{i}^{\prime}\right\|_{\infty} \leq C_{3} \text {. }
$$

3.2. The convergence of the methods. We now give a result concerning the growth of a perturbation in the solution computed in a single step of the method.

Lemma 3.1. Let the assumptions of Theorem 3.1 be satisfied. Suppose that $v_{n}=v\left(x_{n}\right)+e_{n}$, where $\left\|e_{n}\right\|=\mathscr{O}\left(h^{k_{G}}\right)$. Let $v_{n+1}$ be the solution of

$$
\begin{aligned}
& f\left(v_{n}+h \sum_{j=1}^{s} a_{i j} \widetilde{V}_{j}^{\prime}, \widetilde{V}_{i}^{\prime}\right)=0, \\
& g\left(v_{n}+h \sum_{j=1}^{s} a_{i j} \widetilde{V}_{j}^{\prime}\right)=0, \\
& v_{n+1}=v_{n}+h \sum_{i=1}^{s} b_{i} \widetilde{V}_{i}^{\prime} .
\end{aligned}
$$

Then

$$
e_{n+1}=\left(I_{m}-\left(b^{T} \mathscr{A}^{-1} \varepsilon_{s}\right)\left[\begin{array}{l}
f_{v^{\prime}} \\
g_{v}
\end{array}\right]^{-1}\left[\begin{array}{l}
0 \\
g_{v}
\end{array}\right]\right) e_{n}+\tilde{\eta}+d_{n+1},
$$


where

$$
\tilde{\eta}= \begin{cases}\mathscr{O}\left(h^{k_{G}+1}\right) & \text { if } k_{G} \geq 2, \\ \mathscr{O}\left(h^{k_{G}+1}\right) & \text { if } k_{G}=1 \text { and } f \text { is linear in } v^{\prime}, \\ \mathscr{O}(h) & \text { if } k_{G}=1 \text { and } f \text { is nonlinear in } v^{\prime},\end{cases}
$$

and $d_{n+1}$ is the local truncation error.

Proof. Let $\tilde{V}_{i}^{\prime}=V_{i}^{\prime}+\Delta V_{i}^{\prime}$, where $V_{i}^{\prime}$ is the solution of (3.14) if $v_{n}=v\left(x_{n}\right)$. The equation (3.14) can be written as

$$
\begin{aligned}
& f\left(V_{i}+e_{n}+h \sum_{j=1}^{s} a_{i j} \Delta V_{j}^{\prime}, V_{i}^{\prime}+\Delta V_{i}^{\prime}\right)=0, \\
& g\left(V_{i}+e_{n}+h \sum_{j=1}^{s} a_{i j} \Delta V_{j}^{\prime}\right)=0, \ldots, s,
\end{aligned}
$$

where $V_{i}=v\left(x_{n}\right)+h \sum_{j=1}^{s} a_{i j} V_{j}^{\prime}$. Theorem 3.1 shows that $V_{i}=v\left(x_{n}\right)+\mathscr{O}(h)$ and $V_{i}^{\prime}=v^{\prime}\left(x_{n}\right)+\mathscr{O}(h)$. We assume that (3.14) is solved exactly. From Theorem 3.2 with $\hat{\nu}-\nu=e_{n}$ we have that $\left\|\Delta V_{i}^{\prime}\right\| \leq C h$ for $k_{G} \geq 2$, while $\left\|\Delta V_{i}^{\prime}\right\| \leq C_{3}$ if $k_{G}=1$ (see (3.13)). Let $\left\|\Delta V_{i}^{\prime}\right\| \leq \delta$ and $\left\|e_{n}\right\| \leq \varepsilon$. In the following, if nothing else is said, all functions and their derivatives are evaluated in $\left(x_{n}, v\left(x_{n}\right)\right)$. By expanding (3.15) in a Taylor series around $\left(V_{i}, V_{i}^{\prime}\right)$ we have

$$
f\left(V_{i}, V_{i}^{\prime}\right)+f_{v^{\prime}}\left(V_{i}, V_{i}^{\prime}\right) \Delta V_{i}^{\prime}+\eta_{f}=0,
$$

where $\eta_{f}$ is the sum of higher-order terms, composed of terms of the form

$$
\begin{aligned}
& f_{v} \cdot\left(e_{n}+h \sum_{j=1}^{s} a_{i j} \Delta V_{j}^{\prime}\right), \\
& f_{v v^{\prime}} \cdot\left(e_{n}+h \sum_{j=1}^{s} a_{i j} \Delta V_{j}^{\prime}, \Delta V_{i}^{\prime}\right) \text {, } \\
& f_{v^{\prime} v^{\prime}} \cdot\left(\Delta V_{i}^{\prime}, \Delta V_{i}^{\prime}\right) \text {. }
\end{aligned}
$$

Thus, we find that

$$
\eta_{f}=\mathscr{O}(\varepsilon+h \delta)+\mathscr{O}\left(\varepsilon \delta+h \delta^{2}\right)+\mathscr{O}\left(\delta^{2}\right) .
$$

Using that $f_{v^{\prime}}\left(V_{i}, V_{i}^{\prime}\right)=f_{v^{\prime}}+\mathscr{O}(h)$, we have from $(3.16)$

$$
f_{v^{\prime}} \Delta V_{i}^{\prime}=\mathscr{O}\left(\varepsilon+h \delta+\varepsilon \delta+h \delta^{2}+\delta^{2}\right) .
$$

Similarly,

$$
g\left(V_{i}\right)+\left(g_{v}+\mathscr{O}(h)\right)\left(e_{n}+h \sum_{j=1}^{s} a_{i j} \Delta V_{j}\right)+\eta_{g}=0
$$


where the higher-order term $\eta_{g}$ is composed of terms of the form

$$
g_{v v} \cdot\left(e_{n}+h \sum_{j=1}^{s} a_{i j} \Delta V_{j}^{\prime}\right)^{2}
$$

so that

$$
\eta_{g}=\mathscr{O}\left(\varepsilon^{2}+h \delta \varepsilon+h^{2} \delta^{2}\right)
$$

Thus, we find that

$$
g_{v} \Delta V_{i}^{\prime}=-\frac{1}{h}\left(\sum_{j=1}^{s} d_{i j}\right) g_{v} e_{n}+\mathscr{O}\left(\varepsilon+h \delta+\frac{\varepsilon^{2}}{h}+\delta \varepsilon+h \delta^{2}\right) .
$$

Equations (3.17) and (3.18) can be solved for $\Delta V_{i}^{\prime}$ :

$$
\Delta V_{i}^{\prime}=\left[\begin{array}{c}
f_{v^{\prime}} \\
g_{v}
\end{array}\right]^{-1}\left[\begin{array}{c}
0 \\
-\frac{1}{h} \sum_{j=1}^{s} d_{i j} g_{v}
\end{array}\right] e_{n}+\mathscr{O}\left(\varepsilon+h \delta+\varepsilon \delta+h \delta^{2}+\frac{\varepsilon^{2}}{h}+\delta^{2}\right)
$$

To find a lower bound for $\Delta V_{i}^{\prime}$, we use the same strategy as in [1 and 10]. We have

$$
\left\|\Delta V_{i}^{\prime}\right\| \leq K\left(\frac{\varepsilon}{h}+\varepsilon+h \delta+\varepsilon \delta+h \delta^{2}+\frac{\varepsilon^{2}}{h}+\delta^{2}\right) .
$$

Let $\delta$ be the solution of the equation

$$
\delta=K\left(\frac{\varepsilon}{h}+\varepsilon+h \delta+\varepsilon \delta+h \delta^{2}+\frac{\varepsilon^{2}}{h}+\delta^{2}\right) .
$$

Let $\varepsilon=\mathscr{O}\left(h^{k_{G}}\right)$, where $k_{G} \geq 2$, and solve (3.7) by functional iteration $\delta=G(\delta)$ with the initial value $\delta^{(0)}=k_{1} h^{k_{G}-1}$. Then $\delta^{(1)}=G\left(\delta^{(0)}\right)=\mathscr{O}\left(h^{k_{G}-1}\right)$ and $|\partial G / \partial \delta|=\mathscr{O}(h) \leq 1$ for $h$ sufficiently small. We can now use the Banach Fixed Point Theorem to conclude that the iteration converges to a solution satisfying

$$
\delta=\mathscr{O}\left(h^{k_{G}-1}\right)
$$

for $k_{G} \geq 2$. Inserting this into (3.19), we have

$$
\Delta V_{i}^{\prime}=\left[\begin{array}{c}
f_{v^{\prime}} \\
g_{v}
\end{array}\right]^{-1}\left[\begin{array}{c}
0 \\
-\frac{1}{h} \sum_{j=1}^{s} d_{i j} g_{v}
\end{array}\right] e_{n}+\mathscr{O}\left(h^{k_{(j}}\right) \text { for } k_{G} \geq 2
$$

If $k_{G}=1$, then $\delta=\mathscr{O}(1)$, so that

$$
\Delta V_{i}^{\prime}=\left[\begin{array}{c}
f_{v^{\prime}} \\
g_{v}
\end{array}\right]^{-1}\left[\begin{array}{c}
0 \\
-\frac{1}{h} \sum_{j=1}^{s} d_{i j} g_{v}
\end{array}\right] e_{n}+\mathscr{O}(1) \text { for } k_{G}=1
$$

The term $\mathscr{O}(1)$ comes from terms of the form

$$
f_{v^{\prime} v^{\prime}}\left(\Delta V_{i}^{\prime}, \Delta V_{i}^{\prime}\right) \text {. }
$$


These terms do not appear in problems linear in $v^{\prime}$. We conclude that (3.21) is valid for such problems, even for $k_{G}=1$. Inserting (3.21) or (3.22) into (3.14), we have

$$
\begin{aligned}
v_{n+1} & =v_{n}+h \sum_{i=1}^{s} b_{i}\left(V_{i}^{\prime}+\Delta V_{i}^{\prime}\right) \\
& =v\left(x_{n}\right)+h \sum_{i=1}^{s} b_{i} V_{i}^{\prime}+e_{n}-\sum_{i, j=1}^{s} b_{i} d_{i j}\left[\begin{array}{c}
f_{v^{\prime}} \\
g_{v}
\end{array}\right]^{-1}\left[\begin{array}{c}
0 \\
g_{v}
\end{array}\right] e_{n}+\tilde{\eta},
\end{aligned}
$$

where $\tilde{\eta}=\mathscr{O}\left(h^{k_{G}+1}\right)$ for $k_{G} \geq 2$, or if $k_{G}=1$ and $f$ is linear in $v^{\prime}$. If $k_{G}=1$ and $f$ is nonlinear in $v^{\prime}$, then $\tilde{\eta}=\mathscr{O}(h)$. From

$$
v_{n+1}=v\left(x_{n}+h\right)+d_{n+1}+\left(I_{m}-\left(b^{T} \mathscr{A}^{-1} \varepsilon_{s}\right)\left[\begin{array}{c}
f_{v^{\prime}} \\
g_{v}
\end{array}\right]^{-1}\left[\begin{array}{c}
0 \\
g_{v}
\end{array}\right]\right) e_{n}+\tilde{\eta}
$$

we thus have

$$
e_{n+1}=\left(I_{m}-\left(b^{T} \mathscr{A}^{-1} \varepsilon_{s}\right)\left[\begin{array}{l}
f_{v^{\prime}} \\
g_{v}
\end{array}\right]^{-1}\left[\begin{array}{l}
0 \\
g_{v}
\end{array}\right]\right) e_{n}+\tilde{\eta}+d_{n+1} .
$$

We now consider the global error resulting from repeated use of the method.

Theorem 3.3. Suppose the index 1 problem (1.6) is solved numerically by an s-stage Runge-Kutta method. Assume that

1. $f, g$ are sufficiently differentiable.

2. The initial values satisfy $\left\|v_{0}-v\left(x_{0}\right)\right\| \leq \mathscr{O}\left(h^{k_{G}}\right)$ and $f\left(v_{0}, v_{0}^{\prime}\right)=\mathscr{O}(h)$.

3. (a) $k_{G} \geq 2$ for problems nonlinear in $v^{\prime}$;

(b) $k_{G} \geq 1$ for problems linear in $v^{\prime}$, provided that $f\left(v_{n}, v_{n}^{\prime}\right)=\mathscr{O}(h)$ and $g\left(v_{n}\right)=\mathscr{O}\left(h^{2}\right)$.

4. The local truncation error satisfies

(a) $P_{n} d_{n+1}=\mathscr{O}\left(h^{k_{G}+1}\right), S_{n} d_{n+1}=\mathscr{O}\left(h^{k_{G}}\right)$ if $\left|1-b^{T} \mathscr{A}^{-1} \varepsilon_{s}\right|<1$;

(b) $P_{n} d_{n+1}=\mathscr{O}\left(h^{k_{G}+1}\right), S_{n} d_{n+1}=\mathscr{O}\left(h^{k_{G}+1}\right)$ if $\left|1-b^{T} \mathscr{A}^{-1} \varepsilon_{s}\right|=1$, where

$$
S_{n}=\left[\begin{array}{c}
f_{v^{\prime}}\left(v_{n}, v_{n}^{\prime}\right) \\
g_{v}\left(v_{n}\right)
\end{array}\right]^{-1}\left[\begin{array}{c}
0 \\
g_{v}\left(v_{n}\right)
\end{array}\right], \quad P_{n}=I_{m}-S_{n} .
$$

Then the global error is at least of order $k_{G}$.

Before we prove this theorem, we comment on assumptions 2 and 3(b). The relations

$$
f\left(v_{n}, v_{n}^{\prime}\right)=\mathscr{O}(h) \text { and } g\left(v_{n}\right)=\mathscr{O}\left(h^{2}\right)
$$

have to be satisfied to ensure a solution of the system (1.2). The relations (3.23) hold for the initial step by assumption 2 . For $k_{G} \geq 2$, (3.23) is satisfied if $v_{n}^{\prime}=v^{\prime}(x)+\mathscr{O}(h)$, which is easily obtained, for example by using $v_{n+1}^{\prime}=V_{s}^{\prime}$. 
For $k_{G}=1$ we have to make these assumptions explicitly. All Runge-Kutta methods with $b_{i}=a_{s i}$ satisfy assumption (3.23).

Proof of Theorem 3.3. The interval of integration, $\left[x_{0}, x_{\text {end }}\right]$, can be split in $N$ subintervals, $H_{k}=\left[X_{k}, X_{k+1}\right], k=0, \ldots, N-1, X_{0}=x_{0}$, and $X_{N}=x_{\text {end }}$. We assume each $X_{k}$ to coincide with some $x_{n}$. In each subinterval, there exists a constant permutation matrix $Q(x), Q(x) v=\left[y^{T}, z^{T}\right]^{T}$ ensuring $g_{z}$ to be nonsingular over each subinterval. The subintervals are chosen so that $Q(x)$ has to be changed over two adjacent subintervals. First, we will prove the theorem over the first subinterval, $H_{0}$, and we assume the variables to be numbered such that $Q(x)=I_{m}$ in $H_{0}$. Thus, we have

$$
\begin{aligned}
{\left[\begin{array}{l}
f_{v^{\prime}} \\
g_{v}
\end{array}\right]^{-1}=} & {\left[\begin{array}{ll}
f_{y^{\prime}} & f_{z^{\prime}} \\
g_{y} & g_{z}
\end{array}\right]^{-1} } \\
\quad= & {\left[\begin{array}{cc}
\left(f_{y^{\prime}}-f_{z^{\prime}} g_{z}^{-1} g_{y}\right)^{-1} & -\left(f_{y^{\prime}}-f_{z^{\prime}} g_{z}^{-1} g_{y}\right)^{-1} f_{z^{\prime}} g_{z}^{-1} \\
-g_{z}^{-1} g_{y}\left(f_{y^{\prime}}-f_{z^{\prime}} g_{z}^{-1} g_{y}\right)^{-1} & g_{z}^{-1} g_{y}\left(f_{y^{\prime}}-f_{z^{\prime}} g_{z}^{-1} g_{y}\right)^{-1} f_{z^{\prime}} g_{z}^{-1}+g_{z}^{-1}
\end{array}\right] . }
\end{aligned}
$$

Inserting this into the expression for the global error in Lemma 3.1, we have

$$
e_{n+1}=\left(I_{m}-\left(b^{T} \mathscr{A}^{-1} \varepsilon_{s}\right) S(y, z)\right) e_{n}+\tilde{\eta}_{n+1}+d_{n+1},
$$

where

$$
S(y, z)=\left[\begin{array}{cc}
-F_{P} f_{z^{\prime}} g_{z}^{-1} g_{y} & -F_{P} f_{z^{\prime}} \\
g_{z}^{-1} g_{y} F_{P} f_{z^{\prime}} g_{z}^{-1} g_{y}+g_{z}^{-1} g_{y} & g_{z}^{-1} g_{y} F_{P} f_{z^{\prime}}+I_{m-r}
\end{array}\right]
$$

and $F_{P}=\left(f_{y^{\prime}}-f_{z^{\prime}} g_{z}^{-1} g_{y}\right)^{-1}$. Thus, (3.24) can be written as

$$
e_{n+1}=P_{n} e_{n}+r S_{n} e_{n}+\tilde{d}_{n+1}
$$

where $P=I_{m}-S, S_{n}=S\left(y_{n}, z_{n}\right), P_{n}=P\left(y_{n}, z_{n}\right), \tilde{d}_{n+1}=d_{n+1}+\tilde{\eta}_{n+1}$, and $r=1-b^{T} \mathscr{A}^{-1} \varepsilon_{s}$. For each subinterval, $S_{n}$ and $P_{n}$ are projection operators satisfying $S_{n}^{2}=S_{n}$ and $P_{n}^{2}=P_{n}$. In fact, $P(y, z)$ represents a projection into the tangent space of the surface given by $g(y, z)=0$, because $\left[g_{y}, g_{z}\right] P \equiv 0$. The components of $P$ and $S$ are smooth, so that

$$
\begin{array}{cl}
S_{n+1} S_{n}=S_{n}+\mathscr{O}(h), & P_{n+1} P_{n}=P_{n}+\mathscr{O}(h), \\
S_{n+1} P_{n}=\mathscr{O}(h), & P_{n+1} S_{n}=\mathscr{O}(h) .
\end{array}
$$

Multiplying (3.25) once by $P_{n+1}$ and once by $S_{n+1}$, we obtain

$$
\left[\begin{array}{l}
\left\|P_{n+1} e_{n+1}\right\| \\
\left\|S_{n+1} e_{n+1}\right\|
\end{array}\right] \leq\left[\begin{array}{cc}
1+\mathscr{O}(h) & \mathscr{O}(h) \\
\mathscr{O}(h) & |r|(1+\mathscr{O}(h))
\end{array}\right]\left[\begin{array}{l}
\left\|P_{n} e_{n}\right\| \\
\left\|S_{n} e_{n}\right\|
\end{array}\right]+\left[\begin{array}{l}
\left\|P_{n+1} \tilde{d}_{n+1}\right\| \\
\left\|S_{n+1} \tilde{d}_{n+1}\right\|
\end{array}\right] \text {. }
$$

Suppose that $\left\|P_{n+1} \tilde{d}_{n+1}\right\| \leq D_{p}$ and $\left\|S_{n+1} \tilde{d}_{n+1}\right\| \leq D_{s}$. Then (3.26) can be written as

$$
\left[\begin{array}{l}
\left\|P_{n} e_{n}\right\| \\
\left\|S_{n} e_{n}\right\|
\end{array}\right] \leq A^{n}(h)\left[\begin{array}{l}
\left\|P_{0} e_{0}\right\| \\
\left\|S_{0} e_{0}\right\|
\end{array}\right]+\sum_{i=1}^{n} A^{n-i}(h)\left[\begin{array}{c}
D_{p} \\
D_{s}
\end{array}\right]
$$


where

$$
A(h)=\left[\begin{array}{cc}
1+\mathscr{O}(h) & \mathscr{O}(h) \\
\mathscr{O}(h) & |r|(1+\mathscr{O}(h))
\end{array}\right] .
$$

The matrix $A(h)$ can be diagonalized by a matrix $T(h)$ such that

$$
T(h) A(h) T^{-1}(h)=\left[\begin{array}{cc}
1+\mathscr{O}(h) & 0 \\
0 & |r|(1+\mathscr{O}(h))
\end{array}\right],
$$

where

$$
T(h)=\left[\begin{array}{cc}
1+\mathscr{O}(h) & \mathscr{O}(h) \\
\mathscr{O}(h) & 1+\mathscr{O}(h)
\end{array}\right] .
$$

The inequality (3.27) can be written as

$$
\begin{aligned}
{\left[\begin{array}{l}
\left\|P_{n} e_{n}\right\| \\
\left\|S_{n} e_{n}\right\|
\end{array}\right] \leq } & T^{-1}(h)\left[\begin{array}{cc}
\left(1+K_{1} h\right)^{n} & 0 \\
0 & |r|^{n}\left(1+K_{2} h\right)^{n}
\end{array}\right] T(h)\left[\begin{array}{l}
\left\|P_{0} e_{0}\right\| \\
\left\|S_{0} e_{0}\right\|
\end{array}\right] \\
& +T^{-1}(h) \sum_{i=1}^{n}\left[\begin{array}{cc}
\left(1+K_{1} h\right)^{n-i} & 0 \\
0 & |r|^{n-i}\left(1+K_{2} h\right)^{n-i}
\end{array}\right] T(h)\left[\begin{array}{c}
D_{p} \\
D_{s}
\end{array}\right] .
\end{aligned}
$$

By direct computation we have for $|r|<1$

$$
\left[\begin{array}{l}
\left\|P_{n} e_{n}\right\| \\
\left\|S_{n} e_{n}\right\|
\end{array}\right] \leq\left[\begin{array}{c}
C_{1}\left\|P_{0} e_{0}\right\|+\mathscr{O}(h)\left\|S_{0} e_{0}\right\| \\
\mathscr{O}(h)\left\|P_{0} e_{0}\right\|+C_{2}\left(|r|^{n}+h\right)\left\|S_{0} e_{0}\right\|
\end{array}\right]+\left[\begin{array}{c}
\frac{1}{h} C_{3} D_{p}+C_{4} D_{s} \\
C_{5} D_{p}+C_{6} D_{s}
\end{array}\right]
$$

and for $|r|=1$

$$
\left[\begin{array}{l}
\left\|P_{n} e_{n}\right\| \\
\left\|S_{n} e_{n}\right\|
\end{array}\right] \leq\left[\begin{array}{l}
C_{1}\left\|P_{0} e_{0}\right\|+\mathscr{O}(h)\left\|S_{0} e_{0}\right\| \\
\mathscr{O}(h)\left\|P_{0} e_{0}\right\|+C_{2}\left\|S_{0} e_{0}\right\|
\end{array}\right]+\left[\begin{array}{c}
\frac{1}{h} C_{3} D_{p}+C_{4} D_{s} \\
\frac{1}{h} C_{5} D_{p}+C_{6} D_{s}
\end{array}\right] .
$$

By using the fact that $\left\|e_{n}\right\| \leq\left\|P_{n} e_{n}\right\|+\left\|S_{n} e_{n}\right\|$ we obtain

$$
\left\|e_{n}\right\| \leq \begin{cases}C\left(\left\|P_{0} e_{0}\right\|+\left(|r|^{n}+h\right)\left\|S_{0} e_{0}\right\|+\frac{1}{h} D_{p}+D_{s}\right) & \text { for }|r|<1, \\ C\left(\left\|e_{0}\right\|+\frac{1}{h}\left(D_{p}+D_{s}\right)\right) & \text { for }|r|=1 .\end{cases}
$$

Thus, if $e_{0}=\mathscr{O}\left(h^{k_{G}}\right), D_{s}=\mathscr{O}\left(h^{k_{G}+1}\right)$, and $D_{s}=\mathscr{O}\left(h^{k_{G}}\right)$ for $|r|<1$, or $D_{s}=\mathscr{O}\left(h^{k_{G}+1}\right)$ for $|r|=1$, we have that the global error at the end of the first subinterval is given by

$$
E_{1}=e_{n}=\mathscr{O}\left(h^{k_{(i}}\right) .
$$

In that case, the global error after $k+1$ subintervals is bounded by

$$
\left\|E_{k+1}\right\| \leq G\left\|E_{k}\right\|+D h^{k_{G}}
$$

and the error at the end of the interval of integration is bounded by

$$
\left\|E_{N}\right\| \leq C^{N}\left\|e_{0}\right\|+\left(\sum_{i=1}^{N} C^{N-i} D\right) h^{k_{G}} \text {. }
$$

Theorem 3.4. Let assumptions 1, 2, and 3 of Theorem 3.3 be satisfied. Then, if $\left|1-b^{T} \mathscr{A}^{-1} \varepsilon_{s}\right|<1$, and

$$
\Phi(t)=\frac{1}{\gamma(t)} \quad \forall t \in \begin{cases}\overline{\mathrm{DA} 1 \mathrm{~T}_{y y}}, & \rho(t) \leq k_{G}, \\ \overline{\mathrm{DA}_{1 \mathrm{~T}}}, & \rho(t) \leq k_{G}-1,\end{cases}
$$

the order of the global error is $k_{G}$. 
Proof. We first prove this theorem for the model equation (2.1). The local truncation error for a method with local order $k_{L}$ applied to this problem is given by

$$
d_{n}=\left[\begin{array}{c}
\sum_{\substack{t \in \mathrm{DA}_{1} \mathrm{~T}_{y} \\
\rho(t)=k_{L}}}(\gamma(t) \Phi(t)-1) \alpha(t) F(t) \frac{h^{\rho(t)}}{\rho(t) !} \\
\sum_{\substack{u \in \mathrm{DA}_{\mathrm{I}} \mathrm{T}_{j} \\
\rho(u)=k_{L}}}(\gamma(u) \Phi(u)-1) \alpha(u) G(u) \frac{h^{\rho(u)}}{\rho(u) !}
\end{array}\right]+\mathscr{O}\left(h^{k_{L}+1}\right) .
$$

The first term in this expression is called the principal error term. The principal error term can also be written as

$$
\begin{gathered}
{\left[\begin{array}{cc}
\left(-f_{z^{\prime}} g_{y}\right)^{-1} & -\left(-f_{z^{\prime}} g_{y}\right)^{-1} f_{z^{\prime}} \\
g_{y}\left(-f_{z^{\prime}} g_{y}\right)^{-1} & -g_{y}\left(-f_{z^{\prime}} g_{y}\right)^{-1} f_{z^{\prime}}+I_{n-r}
\end{array}\right]} \\
{\left[\begin{array}{cc}
\sum_{\substack{t \in \mathrm{DAlT}_{w y} \\
\rho(t)=k_{L}}}(\gamma(t) \Phi(t)-1) \alpha(t) \widetilde{F}(t) \frac{h^{\rho(t)}}{\rho(t) !} \\
\sum_{\substack{u \in \mathrm{DAlT}_{i} \\
\rho(u)=k_{L}}}(\gamma(u) \Phi(u)-1) \alpha(u) G(u) \frac{h^{\rho(u)}}{\rho(u) !}
\end{array}\right],}
\end{gathered}
$$

where $\tilde{F}(t)=f_{k y l z^{\prime}}\left(F\left(t_{1}\right), \ldots, F\left(t_{k}\right), G\left(u_{1}\right), \ldots, G\left(u_{l}\right)\right)$ for $t \in\left[t_{1}, \ldots, t_{k}\right.$, $\left.u_{1}, \ldots, u_{l}\right]_{y}$, and $F(t)$ and $G(u)$ are given by Definition 2.8. For the model problem, $S(y, z)$ and $P(y, z)$ are given by

$$
S=\left[\begin{array}{cc}
I_{r} & -\left(-f_{z^{\prime}} g_{y}\right)^{-1} f_{z^{\prime}} \\
0 & I_{m-r}-g_{y}\left(-f_{z^{\prime}} g_{y}\right)^{-1} f_{z^{\prime}}
\end{array}\right], \quad P=\left[\begin{array}{cc}
0 & \left(-f_{z^{\prime}} g_{y}\right)^{-1} f_{z^{\prime}} \\
0 & g_{y}\left(-f_{z^{\prime}} g_{y}\right)^{-1} f_{z^{\prime}}
\end{array}\right] .
$$

Multiplying $d_{n}$ by $P_{n}$, we obtain

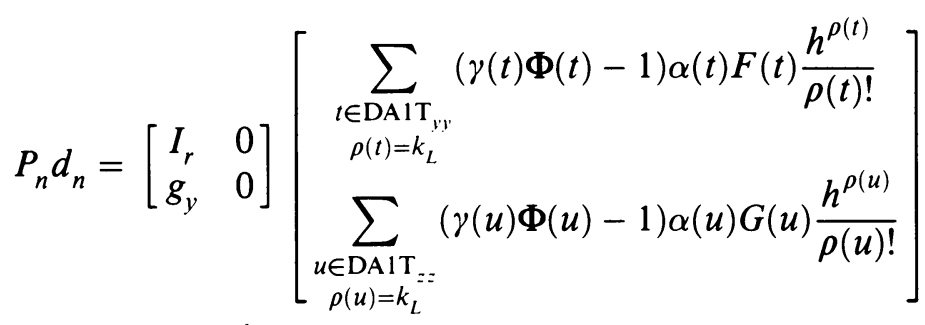

$$
\begin{aligned}
& +\mathscr{O}\left(h^{k_{L}+1}\right) \text {. }
\end{aligned}
$$

Let $\Phi(t)=1 / \gamma(t)$ for all $t \in \mathrm{DAlT}_{y y}, \rho(t)=k_{L}$, and $\Phi(t) \neq 1 / \gamma(t)$ for at least one $t \in \mathrm{DA}_{\mathrm{DT}}, \rho(t)=k_{L}$. By (3.30) we have that $P_{n} d_{n}=\mathscr{O}\left(h^{k_{L}+1}\right)$, while $S_{n} d_{n}=\mathscr{O}\left(h^{k_{L}}\right)$. From Theorem 3.3 we then know that the global order of the method is $k_{L}$. Now, the assertion of the theorem follows directly from 
the fact that the set of simplified trees associated to DA1T $\mathrm{T}_{y y}$, resp. DA1T $z$, is $\overline{\mathrm{DA}_{1 \mathrm{~T}} y y}$, resp. $\overline{\mathrm{DA}_{1 \mathrm{~T}}}$.

This result can be extended also to include the more general equation (1.6). For this equation, the principal error is composed of terms of the form

$$
\left[\begin{array}{cc}
f_{y^{\prime}} & f_{z^{\prime}} \\
g_{y} & g_{z}
\end{array}\right]^{-1}\left[\begin{array}{c}
f_{k y l z k y^{\prime} \hat{l}^{\prime}}\left(y^{\left(p_{1}\right)}, \ldots, y^{\left(p_{k+k}\right)}, z^{\left(q_{1}\right)}, \ldots, z^{\left(q_{l+i}\right)}\right) \\
g_{k y l z}\left(y^{\left(p_{1}\right)}, \ldots, y^{\left(p_{k}\right)}, z^{\left(q_{1}\right)}, \ldots, z^{\left(q_{k}\right)}\right)
\end{array}\right] h^{k_{L}},
$$

where $y^{(p)}$ and $z^{(q)}$ are derivatives of $y$ and $z$ of order less than $k_{L}$. We can show that

$$
P\left[\begin{array}{ll}
f_{y^{\prime}} & f_{z^{\prime}} \\
g_{y} & g_{z}
\end{array}\right]^{-1}=\left[\begin{array}{cc}
\left(f_{y^{\prime}}-f_{z^{\prime}} g_{z}^{-1} g_{y}\right)^{-1} & 0 \\
-g_{z}^{-1} g_{y}\left(f_{y^{\prime}}-f_{z^{\prime}} g_{z}^{-1} g_{y}\right)^{-1} & 0
\end{array}\right] .
$$

The projection operator $P$ suppresses all the contributions to the local truncation error coming from derivatives of the algebraic equation. Since the algebraic equation in (1.6) and the model problem are equivalent, assuming $g_{z}$ nonsingular in (1.6), the conclusion for the model equation is also valid for the general problem.

\section{NUMERICAL EXPERIMENTS}

In this section we present the results of some numerical experiments on various index 1 systems. The experiments confirm that the local order predicted in $\S 2$ occurs in practice, and in no case is the observed global order less than the lower bound given in Theorems 3.3 and 3.4. The experiments below were performed in single precision on a Cray X-MP computer. The test problems are:

P1: A linear constant-coefficient system of the form $A v^{\prime}+B v=g(x)$.

P2: A linear system with time-dependent coefficients, $A(x) v^{\prime}+B(x) v=$ $g(x)$.

P3: A nonlinear system, linear in $v^{\prime}, A(v, x) v^{\prime}=f(v, x)$.

P4: A system nonlinear also in $v^{\prime}, f\left(v, v^{\prime}, x\right)=0$.

The problems are described in Appendix A. The test problems were solved by the following Runge-Kutta methods:

M1: 2-stage, 3rd-order, $A$-stable SDIRK method (Nørsett [11]).

M2: 3-stage, 3rd-order, $B$-stable SDIRK method (Nørsett and Thomsen [12]).

M3: 5-stage, 4th-order, strongly $S$-stable SDIRK method (Cash [4]).

M4: 7-stage, 3rd-order, extrapolation method, based on fully implicit backward Euler, written as a DIRK method.

M5: 2-stage, 2nd-order, Lobatto IIIC method (Chipman [5]).

M6: 3-stage, 4th-order, Lobatto IIIC method (Chipman [5]).

M7: 3-stage, 5th-order, Radau IA method (Butcher [3, p. 228]).

M8: 2-stage, 4th-order, Kuntzmann-Butcher method (Butcher [3, p. 219]). 
M9: 3-stage, 6th-order, Kuntzmann-Butcher method (Butcher [3, p. 220]).

The results of the experiments are given in Tables 4.1-4.4. The following notations have been used:

$k_{d}$ : The order of the method when applied to an ODE.

$k_{P}$ : The order of the global error, predicted by Burrage and Petzold in [2, Theorem 1].

$k_{L}$ : Predicted order of the local error.

$k_{l}$ : Observed order of the local error.

$k_{G}$ : Predicted order of the global error.

$k_{g}$ : Observed order of the global error.

TABle 4.1. Predicted/Observed Orders for P1.

\begin{tabular}{|c|c|c|c|c|c|c|}
\hline Method & $k_{d}$ & $k_{P}$ & $k_{L}$ & $k_{l}$ & $k_{G}$ & $k_{g}$ \\
\hline M1 & 3 & 2 & 2 & 2 & 2 & 2 \\
M2 & 3 & 2 & 2 & 2 & 2 & 2 \\
M3 & 4 & 2 & 3 & 5 & 2 & 4 \\
M4 & 3 & 2 & 4 & 4 & 3 & 3 \\
M5 & 2 & 2 & 3 & 3 & 2 & 2 \\
M6 & 4 & 3 & 5 & 5 & 4 & 4 \\
M7 & 5 & 3 & 3 & 3 & 3 & 3 \\
M8 & 4 & 2 & 3 & 3 & 2 & 2 \\
M9 & 6 & 4 & 4 & 4 & 3 & 4 \\
\hline
\end{tabular}

TABle 4.2. Predicted/Observed Orders for P2.

\begin{tabular}{|c|c|c|c|c|c|c|}
\hline Method & $k_{d}$ & $k_{P}$ & $k_{L}$ & $k_{l}$ & $k_{G}$ & $k_{g}$ \\
\hline M1 & 3 & 2 & 2 & 2 & 2 & 2 \\
M2 & 3 & 2 & 2 & 2 & 2 & 2 \\
M3 & 4 & 2 & 3 & 5 & 2 & 4 \\
M4 & 3 & 2 & 4 & 4 & 3 & 3 \\
M5 & 2 & 2 & 3 & 3 & 2 & 2 \\
M6 & 4 & 3 & 5 & 5 & 4 & 4 \\
M7 & 5 & 3 & 3 & 3 & 3 & 3 \\
M8 & 4 & 2 & 3 & 3 & 2 & 2 \\
M9 & 6 & 4 & 4 & 4 & 3 & 4 \\
\hline
\end{tabular}

TABle 4.3. Predicted/Observed Orders for P3.

\begin{tabular}{|c|c|c|c|c|c|c|}
\hline Method & $k_{d}$ & $k_{P}$ & $k_{L}$ & $k_{l}$ & $k_{G}$ & $k_{g}$ \\
\hline M1 & 3 & 2 & 2 & 2 & 2 & 2 \\
M2 & 3 & 2 & 2 & 2 & 2 & 2 \\
M3 & 4 & 2 & 3 & 3 & 2 & 2 \\
M4 & 3 & 2 & 4 & 4 & 3 & 3 \\
M5 & 2 & 2 & 3 & 3 & 2 & 2 \\
M6 & 4 & 3 & 5 & 5 & 4 & 4 \\
M7 & 5 & 3 & 3 & 3 & 3 & 3 \\
M8 & 4 & 2 & 3 & 3 & 2 & 2 \\
M9 & 6 & 4 & 4 & 4 & 3 & 4 \\
\hline
\end{tabular}


TABle 4.4. Predicted/Observed Orders for P4.

\begin{tabular}{|c|c|c|c|c|c|c|}
\hline Method & $k_{d}$ & $k_{P}{ }^{\mathrm{a}}$ & $k_{L}$ & $k_{l}$ & $k_{G}$ & $k_{g}$ \\
\hline M1 & 3 & - & 2 & 2 & 2 & 2 \\
M2 & 3 & - & 2 & 2 & 2 & 2 \\
M3 & 4 & - & 3 & 3 & 2 & 2 \\
M4 & 3 & - & 4 & 4 & 3 & 3 \\
M5 & 2 & - & 3 & 3 & 2 & 2 \\
M6 & 4 & - & 5 & 5 & 4 & 4 \\
M7 & 5 & - & 3 & 3 & 3 & 3 \\
M8 & 4 & - & 3 & 3 & 2 & 2 \\
M9 & 6 & - & 4 & 4 & 3 & 4 \\
\hline
\end{tabular}

${ }^{\mathrm{a}}$ Theorem 1 in [2] gives no lower bound for problems nonlinear in $v^{\prime}$.

In no case is the observed local and global order lower than the predicted one. However, for P1, the order observed is higher than expected for several methods. The reason is the simplicity of the problem. For linear, constantcoefficients systems, the only order conditions which have to be satisfied, in addition to the classical ODE-conditions, are

$$
b^{T} \mathscr{A}^{-1} c^{j}=1, \quad j=1, \ldots, q,
$$

where $q=k_{G}-1$ if $|r|<1$, or $q=k_{G}$ if $|r|=1$. The Cash method M3 solves problem $\mathrm{P} 2$ with a higher than expected order for similar reasons. The elementary differentials causing the method to be reduced to an order- 2 method are not present in this problem.

For the rest of the problems, there is agreement between the observed and predicted local order. There is also agreement between the observed and predicted global order, with one exception, the Kuntzmann-Butcher method M9. In this case, the stability constant $r=1-b^{T} \mathscr{A}^{-1} \varepsilon_{s}=-1$. The contribution to the global error from the local error of two adjacent steps is $d_{n+1}+r d_{n}=\mathscr{O}\left(h^{k_{L}+1}\right)$, that is, the local errors from two adjacent steps cancel each other. See [2] for a better explanation of this phenomenon. M9 is also the only method where the order predicted by Burrage and Petzold is better than the order predicted by our theory.

\section{Discussion}

In this paper we have derived a set of necessary and sufficient order conditions for the local truncation error when a Runge-Kutta method is applied to a fully implicit differential-algebraic equation of index-1. The numerical experiments, described in $\S 4$, confirm our results.

In Appendix B, the results given in this paper are compared with the results given by Burrage and Petzold [2,13]. We observe that there is no conflict between the two theories. Let $Q(x)$ be a permutation matrix, ensuring 
$g_{z}$ to be nonsingular. The algebraic part of the equation is $g(v)=0$, and $v=Q^{-1}(x)\left[y^{T}, z^{T}\right]^{T}$. The order conditions derived in $\S 2$ are only valid if $Q(x)$ does not change over the step. The theory of Petzold does not have this restriction.

If $Q(x)$ does not vary too frequently, it is possible to use embedding for the control of the local error. In practice, one should be careful when choosing a method for solving general classes of index-1 equations. Some methods will attain a higher order for some classes of equations, like semiexplicit equations and linear constant-coefficient equations. This is observed in $\S 4$, Table 4.1. For the linear constant-coefficient problem, the two variables were solved with different accuracy. For the methods with $r=0, v_{1}$ was solved with order $k_{d}$, while $v_{2}$ was solved exactly. When choosing a method for solving DAE's, such aspects have to be considered.

\section{ACKNOWLEDGMENTS}

The author thanks her thesis adviser, Syvert P. Nørsett, for encouragement and guidance. She is also grateful to Michel Roche and the unknown referees for their suggestions, which improved the original convergence results.

\section{APPENDIX A. TEST PROBLEMS}

\section{P1: Linear constant-coefficient problem.}

$$
\left[\begin{array}{ll}
1 & 2 \\
2 & 4
\end{array}\right] v^{\prime}+\left[\begin{array}{ll}
1 & 2 \\
2 & 5
\end{array}\right] v=\left[\begin{array}{c}
0 \\
\sin x
\end{array}\right]
$$

with $x \in[0,1]$ and initial values

$$
v(0)=\left[\begin{array}{l}
1 \\
0
\end{array}\right] \quad \text { and } \quad v^{\prime}(0)=\left[\begin{array}{c}
-3 \\
1
\end{array}\right]
$$

The exact solution is

$$
v_{1}(x)=e^{-x}-2 \sin x, \quad v_{2}(x)=\sin x .
$$

The local order was derived at $x=0.5$.

P2: Linear problem with time-dependent coefficients.

$$
\begin{gathered}
(x+1) v_{1}^{\prime}+(x+1) v_{2}^{\prime}+x v_{1}-0.5 v_{2}=e^{-x} \\
\left(x^{2}-1.3^{2}\right) v_{1}+\left(x^{2}-0.3^{2}\right) v_{2}=\left(x^{2}-1.3^{2}\right) x e^{-x}+\left(x^{2}-0.3^{2}\right) \sqrt{x+1}
\end{gathered}
$$

with $x \in[0,1]$ and initial values

$$
v(0)=\left[\begin{array}{l}
0 \\
1
\end{array}\right] \quad \text { and } \quad v^{\prime}(0)=\left[\begin{array}{c}
1 \\
0.5
\end{array}\right]
$$

The exact solution is

$$
v_{1}(x)=x e^{-x}, \quad v_{2}(x)=\sqrt{x+1}
$$

The local order was derived at $x=0.28$. 
P3: Nonlinear problem, linear in $v^{\prime}$.

$$
\begin{gathered}
v_{1}^{\prime}+v_{3} v_{2}^{\prime}-\left(v_{2}+1\right) v_{3}^{\prime}=-v_{1}+1+\sin x, \\
\left(v_{3}+1\right) v_{1}^{\prime}+v_{1} v_{2}^{\prime}=-e^{-x}, \\
0=v_{1} v_{2} v_{3}-0.5 e^{-x} \sin (2 x)
\end{gathered}
$$

with $x \in[0,1]$ and initial values

$$
v(0)=\left[\begin{array}{l}
1 \\
0 \\
1
\end{array}\right] \quad \text { and } \quad v^{\prime}(0)=\left[\begin{array}{c}
-1 \\
1 \\
0
\end{array}\right]
$$

The exact solution is

$$
v_{1}(x)=e^{-x}, \quad v_{2}(x)=\sin x, \quad v_{3}(x)=\cos x
$$

The local order was derived at $x=0.5$.

P4: Problem nonlinear in $v^{\prime}$.

$$
\begin{gathered}
\left(\sin ^{2} v_{1}^{\prime}+\cos ^{2} v_{2}\right)\left(v_{2}^{\prime}\right)^{2}-(x-6)^{2}(x-2)^{2} v_{1} e^{-x}=0 \\
(4-x)\left(v_{2}+v_{1}\right)^{3}-64 x^{2} e^{-x} v_{1} v_{2}=0
\end{gathered}
$$

with $x \in[0.5,1]$. The exact solution is

$$
v_{1}=x^{4} e^{-x}, \quad v_{2}=x^{3} e^{-x}(4-x) \text {. }
$$

The local order was derived at $x=0.75$.

\section{APPENDIX B. COMPARISON BETWEEN OUR RESULTS \\ AND THE ORDER RESUlts GIVEN BY PETzold}

Here we want to explain why there is no contradiction between the order results given by Petzold et al. [2, 13] and the results given in this paper. In fact, we can prove that $k_{G} \geq k_{P}, k_{P}$ is the order predicted by Petzold in the theorem cited below, for all methods with $r \neq-1$. The following relations are defined in [2]:

$$
\begin{array}{ll}
A(w): \sum_{i, j=1}^{s} b_{i} d_{i j} c_{j}^{p}=1, & p=1, \ldots, w, \\
B(w): \sum_{i=1}^{s} b_{i} c_{i}^{p-1}=\frac{1}{p}, & p=1, \ldots, w, \\
C(w): \sum_{j=1}^{s} a_{i j} c_{j}^{p-1}=\frac{c_{i}^{p}}{p}, & i=1, \ldots, s, p=1, \ldots, w .
\end{array}
$$

Theorem 1 in [2] is as follows. 
Theorem 1. Suppose that (1.1) is uniform index 1 and linear in $v^{\prime}$, the RungeKutta method satisfies the stability condition $|r| \leq 1$, the errors in the initial conditions are $\mathscr{O}\left(h^{k_{P}}\right)$, and the errors in terminating the Newton iterations are $\mathscr{O}\left(h^{k_{P}+\delta}\right)$ where $\delta=1$ if $|r|=1$ and $\delta=0$ otherwise, and $k_{P} \geq 2$. Then the global errors satisfy $\left\|e_{n}\right\|=\mathscr{O}\left(h^{k_{P}}\right)$, where

$$
k_{P}= \begin{cases}q & \text { if } C(q) \text { and } B(q), \\ q+1 & \text { if } C(q), B(q+1), \text { and }-1 \leq r<1, \\ q+1 & \text { if } C(q), B(q+1), A(q+1), \text { and } r=1 .\end{cases}
$$

This, together with the following lemma and Theorem 3.3 or Theorem 3.4 shows that $k_{G} \geq k_{P}$, for all methods with $r \neq-1$.

Lemma B.1. If $C(q), B(q)$, then

$$
\Phi(t)=\frac{1}{\gamma(t)} \quad \forall t \in \overline{\mathrm{DA}_{1} \mathrm{~T}_{y}}, \rho(t) \leq q .
$$

If $C(q), B(q+1)$, then

$$
\Phi(t)=\frac{1}{\gamma(t)} \quad\left\{\begin{array}{l}
\forall t \in \overline{\mathrm{DA}^{2} \mathrm{~T}_{y y}}, \rho(t) \leq q+1, \\
\forall t \in \overline{\mathrm{DA} 1 \mathrm{~T}_{y z}}, \rho(t) \leq q .
\end{array}\right.
$$

If $C(q), B(q+1), A(q+1)$, then

$$
\Phi(t)=\frac{1}{\gamma(t)} \quad \forall t \in \overline{\mathrm{DA}^{2} \mathrm{~T}_{y}}, \rho(t) \leq q+1 .
$$

Proof. In the following, we use the notation $\hat{t} \in \overline{\mathrm{DA} \mathrm{T}_{y}}$ for the bushy trees, that is, the trees where all the vertices are directly connected to the root. For such trees we have

$$
\mathbf{l}_{i}(\hat{t})=\rho(\hat{t}) c_{i}^{\rho(\hat{t})-1}, \quad i=1, \ldots, s .
$$

Also, $\tau_{y}$ is a bushy tree. Similarly, we use the notation $\hat{u}$ for trees composed by only bushy trees, that is,

$$
\hat{u}=\left[\hat{t}_{1}, \ldots, \hat{t}_{k}\right]_{z} .
$$

Now from (2.34) and (B.1) we have

$$
\mathbf{k}_{i}(\hat{u})=\sum_{j=1}^{s} d_{i j} \sum_{n_{1}, \ldots, n_{k}=1}^{s} \rho\left(\hat{t}_{1}\right) a_{j n_{1}} c_{n_{1}}^{\rho\left(\hat{t}_{1}\right)-1} \cdots \rho\left(\hat{t}_{k}\right) a_{j n_{k}} c_{n_{k}}^{\rho\left(\hat{t}_{k}\right)-1} .
$$

If $C(q)$, where $q+1 \geq \rho(\hat{u})$, this equation can be written as

$$
\mathbf{k}_{i}(\hat{u})=\sum_{j=1}^{s} d_{i j} c_{j}^{\rho\left(\hat{t}_{1}\right)} \cdots c_{j}^{\rho\left(\hat{t}_{k}\right)}=\sum_{j=1}^{s} d_{i j} c_{j}^{\rho(\hat{u})},
$$

where the fact has been used that $\rho(\hat{u})=\rho\left(\hat{t}_{1}\right)+\cdots+\rho\left(\hat{t}_{k}\right)$. If $q \geq \rho(\hat{u})$, then

$$
\mathbf{k}_{i}(\hat{u})=\rho(\hat{u}) c_{i}^{\rho(\hat{u})-1} .
$$


Now let $t=\left[\hat{t}_{1}, \ldots, \hat{t}_{k}, \hat{u}_{1}, \ldots, \hat{u}_{l}\right]_{y} \in \overline{\mathrm{DA} 1 \mathrm{~T}}_{y y}$. Then, from (2.33) and (B.3), we have

$$
\mathbf{l}_{i}(t)=\rho(t) \sum_{n_{1}, \ldots, n_{k}=1}^{s} \rho\left(\hat{t}_{1}\right) a_{i n_{1}} c_{n_{1}}^{\rho\left(\hat{t}_{1}\right)-1} \cdots \rho\left(\hat{t}_{k}\right) a_{i n_{k}} c_{n_{k}}^{\rho\left(\hat{t}_{k}\right)-1} c_{i}^{\rho\left(\hat{u}_{1}\right)-1} \cdots c_{i}^{\rho\left(\hat{u}_{1}\right)-1} .
$$

If $C(q)$, where $q+1 \geq \rho(t)$, then

$$
\mathbf{l}_{i}(t)=\rho(t) c_{i}^{\rho\left(\hat{t}_{1}\right)} \cdots c_{i}^{\rho\left(\hat{t}_{k}\right)} c_{i}^{\rho\left(\hat{u}_{1}\right)-1} \cdots c_{i}^{\rho\left(\hat{u}_{l}\right)-1}=\rho(t) c_{i}^{\rho(t)-1},
$$

since $\rho(t)=\rho\left(\hat{t}_{1}\right)+\cdots+\rho\left(\hat{t}_{k}\right)+\rho\left(\hat{u}_{1}\right)+\cdots+\rho\left(\hat{u}_{l}\right)-l+1$. By repeated use of $(\mathrm{B} .2)$ and (B.4) we know that if $C(q)$, then for all $t \in \overline{\mathrm{DAlT}}_{y y}$ with $\rho(t) \leq q+1$ we have $\mathbf{l}_{i}(t)=\mathbf{l}_{i}(\hat{t})$, where $\hat{t}$ is a bushy tree, and $\rho(t)=\rho(\hat{t})$. Inserting this into (2.31), we have

$$
\mathbf{y}_{1}(t)=\sum_{i=1}^{s} b_{i} \mathbf{l}_{i}(t)=\rho(t) \sum_{i=1}^{s} b_{i} c_{i}^{\rho(t)-1} \quad \forall t \in \overline{\mathrm{DA}^{(1 T}}, \rho(t) \leq q+1 .
$$

Similarly, for all $t \in \overline{\mathrm{DA} \mathrm{T}_{y z}}, \rho(t) \leq q+1$, there exists a $\hat{u}$ such that $\mathbf{l}_{i}(t)=$ $\mathbf{k}_{i}(\hat{u})$ and $\rho(t)=\rho(\hat{u})$. From (B.2), (B.3), and (2.31) we have

$$
\mathbf{y}_{1}(t)=\sum_{i=1}^{s} b_{i} \mathbf{k}_{i}(\hat{u})=\sum_{i=1}^{s} b_{i} d_{i j} c_{j}^{\rho(t)} \quad \forall t \in \overline{\mathrm{DA} 1 \mathrm{~T}_{y z}}, \rho(t) \leq q+1,
$$

and

$$
\mathbf{y}_{1}(t)=\rho(t) \sum_{i=1}^{s} b_{i} c_{i}^{\rho(t)-1} \quad \forall t \in \overline{\mathrm{DA}^{2} \mathrm{~T}_{y y}}, \rho(t) \leq q+1 .
$$

If $C(q)$, all the order conditions up to order $q+1$ are reduced to $A(q+1)$ and $B(q+1)$. Thus, the lemma is proved.

\section{BIBLIOGRAPHY}

1. K. E. Brenan and L. R. Petzold, The numerical solution of higher index differential/algebraic equations by implicit Runge-Kutta methods, Preprint, UCRL-95906, Lawrence Livermore National Laboratory, 1986.

2. K. Burrage and L. Petzold, On order reduction for Runge-Kutta methods applied to differential/algebraic systems and to stiff systems of ODEs, Preprint, UCRL-98046, Lawrence Livermore National Laboratory, 1988.

3. J. C. Butcher, The numerical analysis of ordinary differential equations, Runge-Kutta and general linear methods, Wiley, New York, 1987.

4. J. R. Cash, Diagonally implicit Runge-Kutta formulae with error estimates, J. Inst. Math. Appl. 24 (1979), 293-301.

5. F. H. Chipman, A-stable Runge-Kutta processes, BIT 11 (1971), 384-388.

6. C. W. Gear, Differential-algebraic equation index transformations, Preprint, Dept. of Computer Science, University of Illinois at Urbana-Champaign, 1986.

7. E. Hairer, Ch. Lubich, and M. Roche, The numerical solution of differential-algebraic systems by Runge-Kutta methods, Report, Dept. de Mathématiques, Université de Genève, 1988.

8. B. Leimkuhler, L. R. Petzold, and C. W. Gear, On the consistent initialization of differential-algebraic equations, Dept. of Computer Science, University of Illinois, Urbana, Illinois, 1987. 
9. A. Kvaernø, Order conditions for Runge-Kutta methods applied to differential-algebraic systems of index 1, Report No. 4/87, Div. of Numerical Mathematics, The Norwegian Institute of Technology, Norway, 1987.

10. P. Lötstedt and L. Petzold, Numerical solution of nonlinear differential equations with algebraic constraints: I Convergence results for backward differentiation formulas, Math. Comp. 46 (1986), 491-516.

11. S. P. Nørsett, Semi explicit Runge-Kutta methods, Report No. 6/74, Div. of Numerical Mathematics, The Norwegian Institute of Technology, Norway, 1974.

12. S. P. Nørsett and P. G. Thomsen, Local error control in SDIRK-methods, BIT 26 (1986), 100-113.

13. L. R. Petzold, Order results for implicit Runge-Kutta methods applied to differential/algebraic system, SIAM J. Numer. Anal. 23 (1986), 837-852.

14. M. Roche, Rosenbrock methods for differential algebraic equations, Numer. Math. 52 (1988), 45-63.

15. __ Implicit Runge-Kutta methods for differential algebraic equations, Report, Dept. de Mathématiques, Université de Genève, 1987.

Division of Mathematical Sciences, The Norwegian Institute of Technology, 7034 TRONDHEIM-NTH, NoRWAY. E-mail: anne@imf.unit.no 